\title{
Insulin decreases atherosclerosis by inducing endothelin receptor $B$ expression
}

\author{
Kyoungmin Park, ${ }^{1}$ Akira Mima, ${ }^{1}$ Qian Li, ${ }^{1}$ Christian Rask-Madsen, ${ }^{1}$ Pingnian He, ${ }^{2}$ \\ Koji Mizutani,, Sayaka Katagiri, ${ }^{1}$ Yasutaka Maeda, ${ }^{1}$ I-Hsien Wu, ${ }^{1}$ Mogher Khamaisi, ${ }^{1}$ \\ Simone Rordam Preil, ${ }^{3}$ Ernesto Maddaloni, ${ }^{1}$ Ditte Sørensen,, ${ }^{1,3,4}$ Lars Melholt Rasmussen, ${ }^{3}$ \\ Paul L. Huang, ${ }^{5}$ and George L. King ${ }^{1}$ \\ 'Dianne Nunnally Hoppes Laboratory Section of Vascular Cell Biology, Joslin Diabetes Center, Harvard Medical School, \\ Boston, Massachusetts, USA. ${ }^{2}$ Department of Cellular and Molecular Physiology, Penn State Hershey College of Medicine, \\ Hershey, Pennsylvania, USA. ${ }^{3}$ Department of Clinical Biochemistry and Pharmacology, Odense University Hospital, \\ University of Southern Denmark, Odense, Denmark. ${ }^{4}$ Danish Diabetes Academy, Odense, Denmark. \\ ${ }^{5}$ Cardiovascular Research Center, Cardiology Division, Massachusetts General Hospital, Harvard Medical School, \\ Charlestown, Massachusetts, USA.
}

Endothelial cell (EC) insulin resistance and dysfunction, caused by diabetes, accelerates atherosclerosis. It is unknown whether specifically enhancing EC-targeted insulin action can decrease atherosclerosis in diabetes. Accordingly, overexpressing insulin receptor substrate-1 (IRS1) in the endothelia of $\mathrm{Apoe}^{-/-}$mice (Irs1/Apoe ${ }^{-/-}$) increased insulin signaling and function in the aorta. Atherosclerosis was significantly reduced in $/ \mathrm{rs} 1 / \mathrm{ApoE}^{-/-}$mice on diet-induced hyperinsulinemia and hyperglycemia. The mechanism of insulin's enhanced antiatherogenic actions in EC was related to remarkable induction of NO action, which increases endothelin receptor B (EDNRB) expression and intracellular [ $\left.\mathrm{Ca}^{2+}\right]$. Using the mice with knockin mutation of eNOS, which had Ser1176 mutated to alanine (AKI), deleting the only known mechanism for insulin to activate eNOS/NO pathway, we observed that IRS1 overexpression in the endothelia of $A$ ki/ApoE ${ }^{-/-}$mice significantly decreased atherosclerosis. Interestingly, endothelial EDNRB expression was selectively reduced in intima of arteries from diabetic patients and rodents. However, endothelial EDNRB expression was upregulated by insulin via P13K/Akt pathway. Finally EDNRB deletion in EC of LdIr/- and Irs1/ Ldlr ${ }^{\prime-}$ mice decreased NO production and accelerated atherosclerosis, compared with $\mathrm{Ldlr}^{-1-}$ mice. Accelerated atherosclerosis in diabetes may be reduced by improving insulin signaling selectively via IRS1/Akt in the EC by inducing EDNRB expression and NO production.

Conflict of interest: The authors have declared that no conflict of interest exists.

Submitted: January 25, 2016 Accepted: April 5, 2016 Published: May 5, 2016

Reference information: JCI Insight. 2016;1(6):e86574. doi:10.1172/jci.insight.86574.

\section{Introduction}

Systemic insulin resistance and its metabolic perturbations can cause vascular dysfunction and accelerate atherosclerosis in people with diabetes and metabolic syndrome (1-3). One suggested mechanism by which diabetes accelerates atherosclerosis is by inhibiting insulin action on the endothelium (4-7). Insulin's major action on the endothelium is to activate endothelial nitric oxide synthase (eNOS) via phosphorylation at Ser1176 by the activation of Akt (8-10). Selective inhibition of insulin-stimulated activation of Akt in endothelial cells (EC) causes reduction in NO and increases in inflammation, leading to endothelial dysfunction, which may accelerate atherosclerosis (7, 10-12). However, it is unknown whether enhancing endothelial insulin action will decrease atherosclerosis, since hyperinsulinemia also correlates with increased risk of cardiovascular disease $(13,14)$

We have proposed that insulin has both antiatherogenic and proatherogenic actions on the vessel wall The antiatherogenic actions are mediated by phosphoinositide-3 kinase (PI3K)/Akt activation (7), leading to phosphorylation/activation of eNOS (p-eNOS) activation (15) and downregulation of adhesion molecules such as VCAM1 (4). In contrast, insulin's proatherogenic actions are mediated mainly by the p-Erk pathway, resulting in migration and proliferation of the vascular smooth muscle cells (VSMC). In insulin resistance, there is selective loss of insulin activation of the endothelial PI3K/Akt/eNOS pathway and acceleration of atherosclerosis $(6,15,16)$. The idea that insulin has antiatherogenic actions is suggested by genetically modified mice, in which insulin receptors were deleted specifically in EC, resulting in enhancement of atherosclerosis (4). To test directly the idea that selective enhancement of insulin action via the 
$\mathrm{PI} 3 \mathrm{~K} /$ Akt pathway can decrease atherosclerosis in insulin resistance and diabetes, we overexpressed insulin receptor substrate-1 (IRS1) in EC of $A p o e^{-/-}$mice to specifically activate the PI3K/Akt/eNOS pathway. This mouse model has decreased atherosclerosis, even in the presence of hyperinsulinemia, and enhanced NO production due to a mechanism of endothelin receptor B (EDNRB) induction by insulin signaling. The clinical relevance of these findings is corroborated by findings that expression of EDNRB is decreased in endothelia of arteries from patients with insulin resistance and diabetes.

\section{Results}

Characterization of IRS1/E $E^{-/-}$mice driven by VE-cadherin promoter. Overexpression of IRS1 was targeted to the endothelium using the VE-cadherin promoter in $\mathrm{Apoe}^{-/-}$mice, deriving selective overexpression of Irs $1^{-/+}$in Apoe ${ }^{-/-}$mice (Irs1/Apoe - $^{-1}$ mice). IRS1 protein and mRNA levels were elevated by 2.6-fold and by 5.8-fold in aortas from Irs1/Apoe ${ }^{-/-}$mice, respectively, whereas IRS1 mRNA expression was not changed in skeletal muscle (Figure 1, A and B). IRS1 mRNA levels were increased in the brains, livers, eyes, and kidneys but not in peripheral blood mononuclear cells or spleens (Supplemental Figure 1; supplemental material available online with this article; doi:10.1172/jci.insight.86574DS1). p-Akt levels were increased by 2.7-fold at basal and 3.2-fold with insulin (10 nM) (Figure 1C), which showed parallel significant increases in tyrosine phosphorylation of IRS1 (Tyr608) (p-IRS1), p-Akt (Ser473), and p-eNOS (Ser1176) in EC from Irs1/Apoe $e^{-1}$ mice versus control (Figure 1, D-F), but p-Erk was not changed (Supplemental Figure 2). p-Akt (Ser473) and p-eNOS (Ser1176) were also elevated in the aortas of Irs1/Apoe $e^{-1-}$ mice versus Apoe $e^{-1-}$ mice at basal and with insulin infusion by 2.1- and 1.7-fold, respectively (Figure 1, G and $\mathrm{H}$ ).

Effect of regular diet, Western diet, or high-fat diet. Since previous reports have shown that Western diet (WD) does not induce substantial hyperinsulinemia in $\mathrm{E}^{-/-}$mice (17), we characterized Irs1/Apoe ${ }^{-/-}$and Apoe $^{-1-}$ mice fed regular diet (RD), WD, or high-fat diet (HFD) for 12 weeks for insulin resistance and hyperglycemia. The weight and fat distribution of Irs $1 / A_{p o e^{-/}}$and $A p o e^{-/}$mice were similar on WD or HFD, with mice on HFD markedly heavier than those on WD and RD (Supplemental Figure 3, A and B). No differences were observed between Irs $1 / \mathrm{Apoe}^{-/-}$mice and Apoe ${ }^{-/-}$mice on WD or HFD in BP (systolic or diastolic), plasma cholesterol, or triglyceride levels (Supplemental Figure 3, C-E), $\mathrm{VO}_{2}, \mathrm{VCO}_{2}$, respiratory exchange ratio, heat, food and water intake, and activities (data not shown). Distribution of cholesterol by lipoprotein density was not different between $A_{p o e^{-/-}}$and Irs I/Apoe $e^{-/-}$mice on WD or HFD, although all mice on HFD had greater elevation of VLDL and LDL levels than mice on WD (Supplemental Figure 3F). The effects of RD, WD, and HFD on systemic insulin resistance studied by i.p. GTT and i.p. ITT were comparable in both types of mice (Figure 2, A and B). Compared with RD, WD and HFD increased blood glucose to diabetic levels in a step-wise manner, with maximum glucose level on HFD reached at 1 hour at $>400 \mathrm{mg} / \mathrm{dl}$. i.p. ITT demonstrated significant increases in insulin resistance in HFD compared with $\mathrm{RD}$ (Figure 2, A and B). Three-fold elevation of blood insulin levels was only observed with HFD in both Apoe $^{-1-}$ and Irs1/Apoe ${ }^{-1-}$ mice (Figure 2C).

Assessment of atherosclerosis. The extent of Sudan IV staining of the aortic root and aorta after 16 weeks of WD or HFD was increased by 12- and 16-fold, respectively, compared with RD-fed Apoe ${ }^{-/}$mice (Figure 2, D and E). Atherosclerotic lesions were decreased by $38 \%$ and $52 \%$ in Irs $1 /$ Apoe $^{-/-}$mice versus Apoe $e^{-1-}$ mice on WD (Supplemental Figure 4) or HFD, respectively (Figure 2, D and E). Serial sections of the aortic root from Irs1/Apoe ${ }^{-/-}$mice (Figure $2 \mathrm{~F}$ and Supplemental Figure 4) showed significant reductions in collagen (50\%), smooth muscle ( $\alpha \mathrm{SMC}$ ) (42\%), and macrophage content (MAC2) (31\%) compared with $E^{-/-}$mice on HFD (Figure 2, F and G). The reduction in atherosclerotic lesions correlated with changes in insulin signaling in aortas of $A p o e^{-/-}$and Irs1/Apoe ${ }^{-/}$mice on RD, WD, and HFD, which showed significant reductions of p-Akt and p-eNOS levels when diets were changed from RD to HFD (Figure 2, H and I). Overexpression of IRS1 significantly enhanced insulin stimulation of p-Akt (1.5- to 1.8-fold) and p-eNOS (1.6- to 2.1-fold) on RD, WD, and HFD (Figure 2, H and I). Since oxidant production can increase in diabetes, even with increased NO production (18), we evaluated nitrotyrosine (NT) levels and dihydroethidium-positive (DHEpositive) fluorescence in the aortas of $A p o e^{-/-}$and Irs1/Apoe ${ }^{-/-}$mice on HFD. NT levels, as assessed by IB analysis (data not shown), were studied in the aortas of $I R S 1 / E^{-/-}$mice fed HFD, which were decreased by $35 \% \pm 8 \%$ as compared with Apoe $e^{-/}$mice (Supplemental Figure 5A). Irs $1 /$ Apoe $^{-/}$mice exhibited significantly lower fluorescence levels of DHE by $46 \% \pm 12 \%$ versus $A p o e^{-/-}$mice (Supplemental Figure 5, B and C).

Regulation of VCAM1 expression and monocyte recruitment. Since VCAM1 expression can affect atherosclerosis (19-21) and be inhibited by insulin (4), we characterized its expressions in EC. The expression of 
A

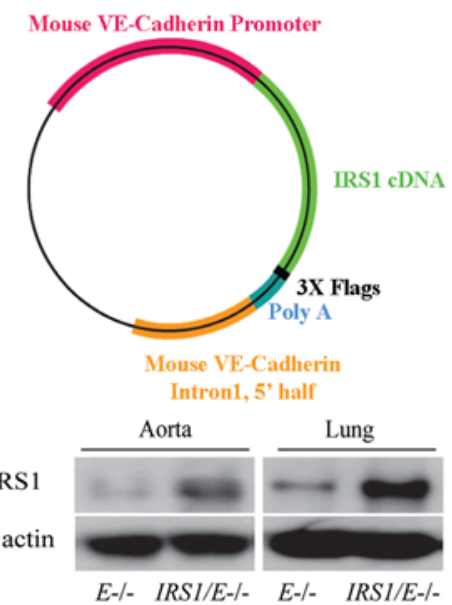

D

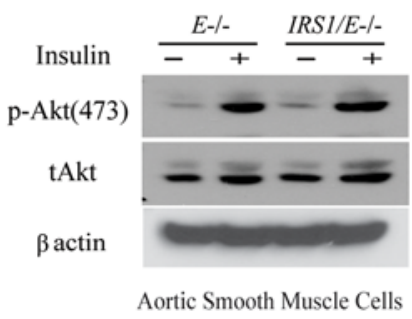

G

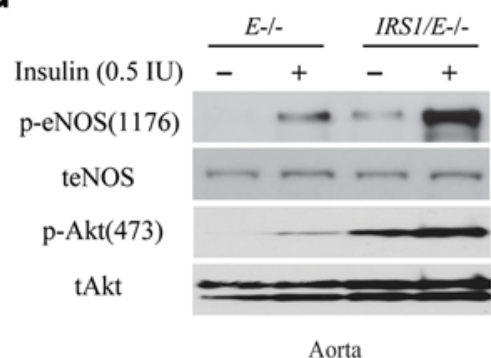

B

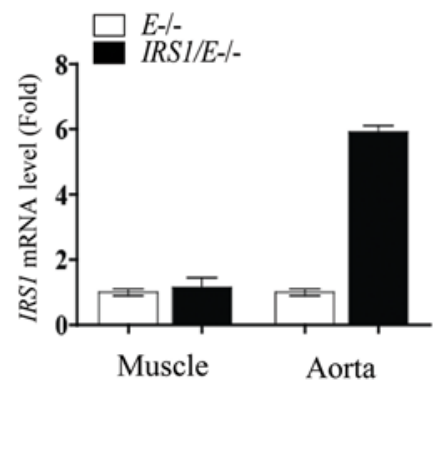

E

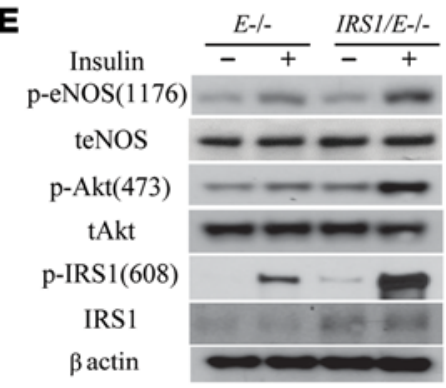

Endothelial Cells
C
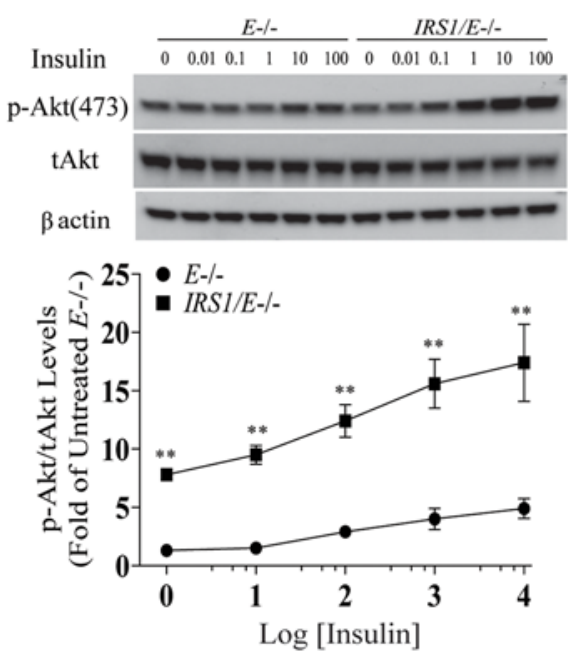

F

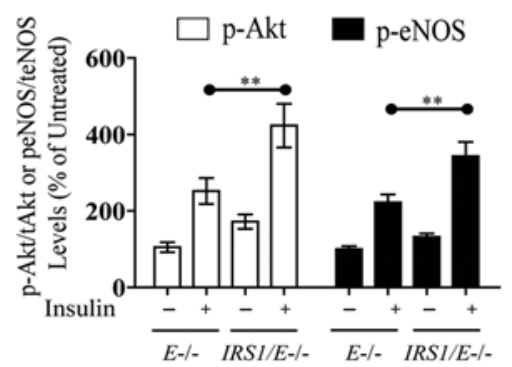

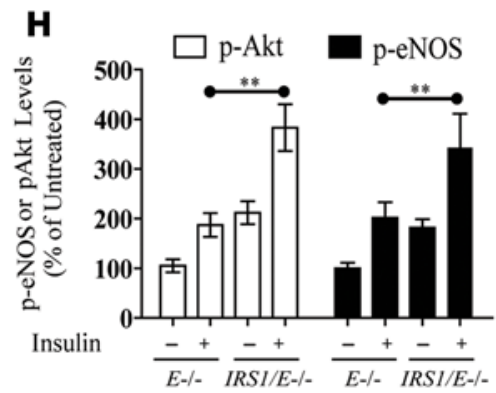

Figure 1. Effect of endothelial overexpression of IRS1 on vascular insulin sensitivity. (A) Schematic representation of IRS1 Tg. IB of IRS1 expression levels in aorta and lung. (B) IRS1 mRNA levels in muscle and aorta. (C) IB and densitometry of p-Akt protein levels in EC after insulin stimulation. (D) IB of p-Akt levels in VSMC. (E and F) IB and densitometry of p-Akt and p-eNOS levels in EC. The IBs are representative of 3 separate experiments that gave similar results. ( $\mathbf{G}$ and $\mathbf{H}$ ) IB and densitometry of $\mathrm{p}$-Akt and $\mathrm{p}$-eNOS protein levels in aortas from Apoe ${ }^{-1-}$ and Irs1/Apoe ${ }^{-/-}$mice treated with insulin. IBs are representative of 3 separate experiments that gave similar results. Data are represented as mean \pm SEM of at least 3 separate experiments. ${ }^{* *} P<0.01(2$-way ANOVA for multiple comparisons involving 2 factorial variables and 2-tailed Student's $t$ test for pairwise comparisons).

VCAM1 protein and VCAM1 mRNA levels was significantly increased by oxidized LDL (ox-LDL) (22), although its elevation was smaller in EC from Irs $1 / \mathrm{Apoe}^{-1-}$ mice versus Apoe ${ }^{-1-}$ mice (Figure 3, A-C). Insulin $(100 \mathrm{nM})$ decreased the expression of VCAM1 significantly in EC from both types of mice and completely normalized VCAM1 mRNA and protein expression in response to ox-LDL in EC from Irs1/Apoe ${ }^{-1-}$ mice in parallel with increased insulin-stimulated activation of p-Akt (Figure 3, A-C). Functionally, ox-LDL enhanced the binding of fluorescent-labeled monocytes to EC from both types of mice, which were inhibited by antibodies to VCAM1 but not control antibodies. Insulin significantly reduced monocyte binding to EC from Irs $1 / \mathrm{Apoe}^{-1-}$ mice by $>45 \%$ versus $\mathrm{Apoe^{-/ }}$ mice (Figure 3D). VCAM1 expression was increased in the aortas of WD- and HFD-fed $A p o e^{-/}$mice versus RD-fed mice (Figure 3, E and F), but its expression was reduced significantly in Irs1/Apoe $e^{-/-}$mice versus Apoe $e^{--}$mice. VCAM1 levels in EC from Irs $1 / A_{p o e^{-1}}$ mice were lower on both WD and HFD in parallel with increased p-Akt (Figure 3, E and F). Analysis of leukocytes in the aortas after 12 weeks on WD showed that macrophages, labeled by antibodies to CD11b, 
A

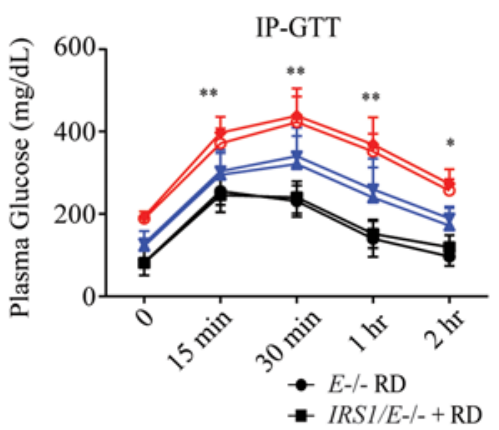

D
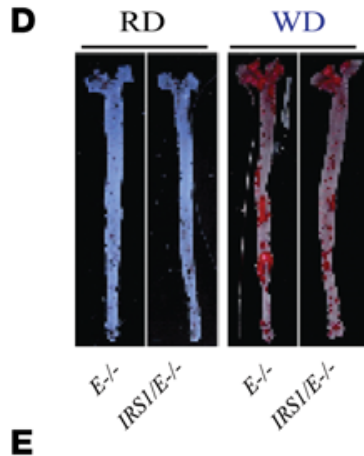

से

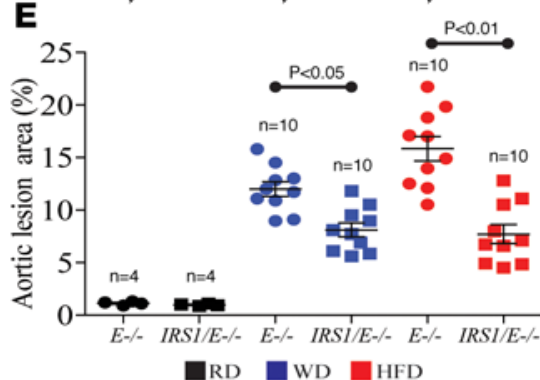

B

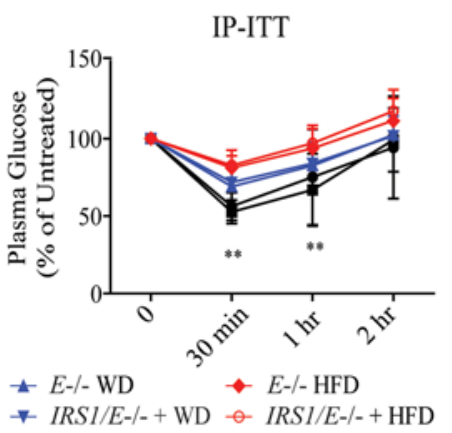

$\mathbf{F}$

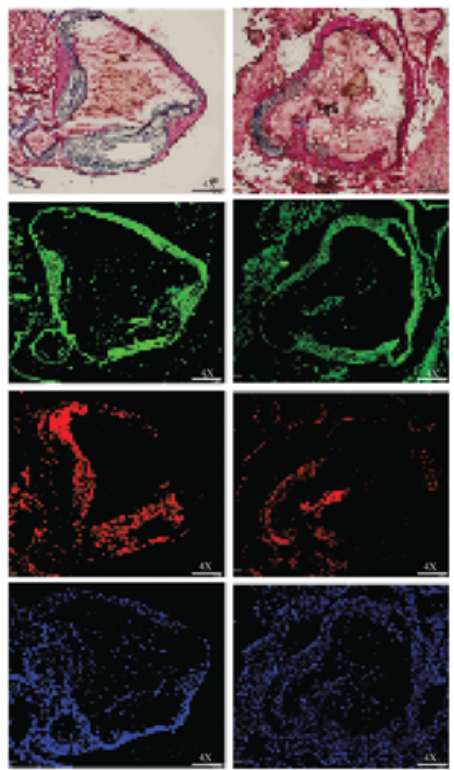

C
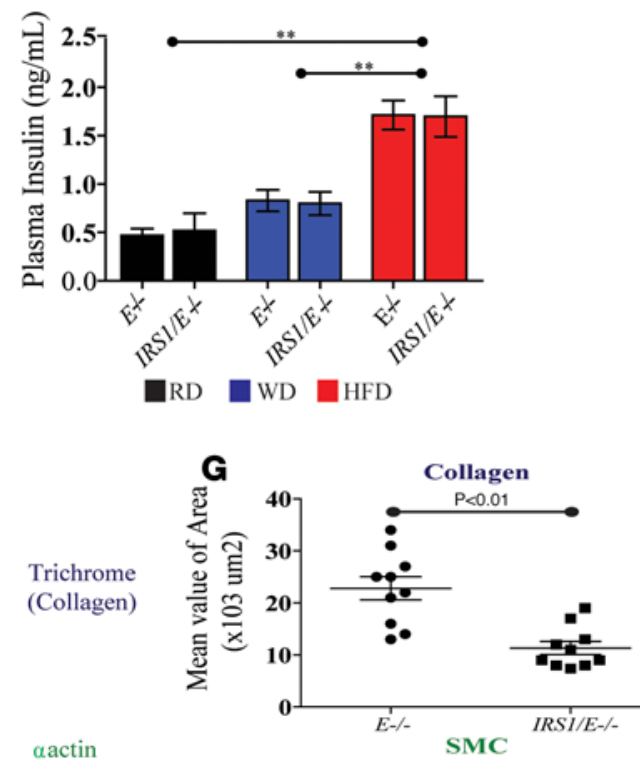

aactin

Trichrome

(Collagen

(SMC)

MAC2

(Macrophage)

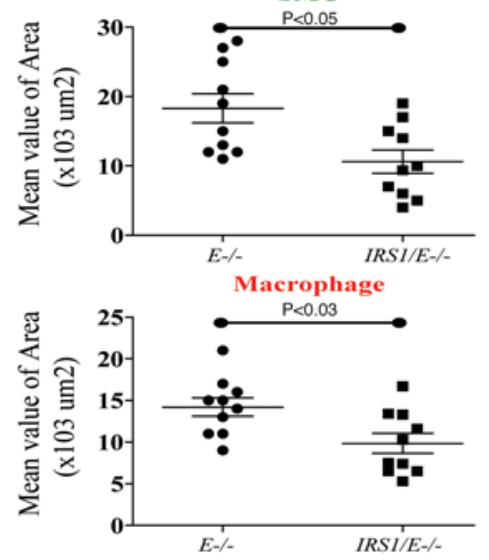

H

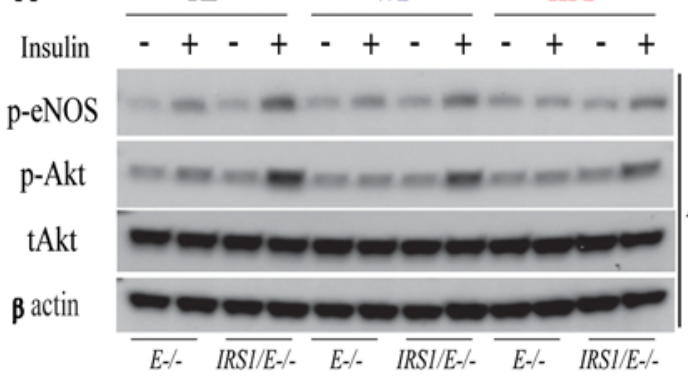

I

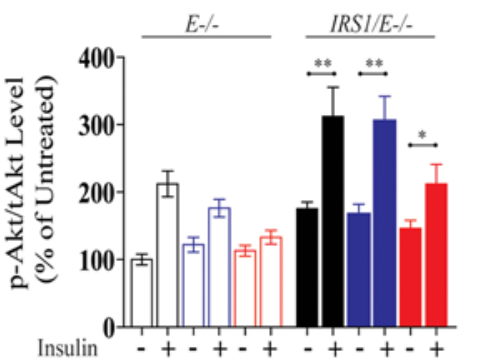

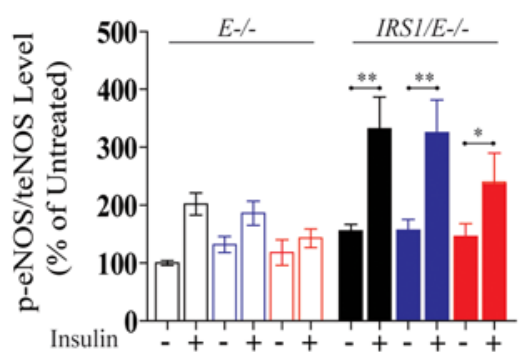

RD $\square$ WD $\square$ HFD

Figure 2. Analysis of the extent of atherosclerosis and its complexity in Irs1/Apoe ${ }^{-/-}$mice fed RD, WD, or HFD. (A) i.p. GTTs were performed in Apoe ${ }^{-/-}$ $(n=8)$ and Irs $1 /$ Apoe $^{-/-}$mice $(n=8)$. Time-course measurements of blood glucose and insulin from the same experiment are shown. This experiment was repeated 3 times in 2 different cohorts of mice. (B) i.p. ITTs were performed in $A p o e^{-/-}(n=8)$ and Irs1/Apoe ${ }^{-/-}$mice $(n=8)$. (C) Fasting insulin in mice fed $\mathrm{RD}, \mathrm{WD}$, or HFD ( $n=8$ per group). $P<0.01$ for main effect of diet. ( $\mathbf{D}$ and $\mathbf{E}$ ) En face staining and quantification of aortas from mice with the indicated genotypes as a percentage of the lesion area in $A_{p o e^{-/-}}(n=10)$ and Irs1/Apoe $e^{-/-}$mice fed HFD $(n=10)$. Original magnification, $\times 2$. (F and $\left.\mathbf{G}\right)$ Representative examples and quantification of cross sections from the aortic sinus stained with trichrome, $\alpha$ actin, and MAC2. Original magnification, $\times 4$; scale bar: 100 $\mu \mathrm{m}$. (H and I) IB and quantification of insulin signaling and p-eNOS in aortas isolated after intravenous insulin injection from mice fed RD, WD, or HFD for 12 weeks ( $n=5$ per group). All data are represented as mean \pm SEM of at least 5 mouse replicates. ${ }^{*} P<0.05$, ${ }^{* *} P<0.01$ (mixed effects model for repeated measurement; 2-way/3-way ANOVA for multiple comparisons involving 2/3 factorial variables and 2-tailed Student's $t$ test for pairwise comparisons). 
A

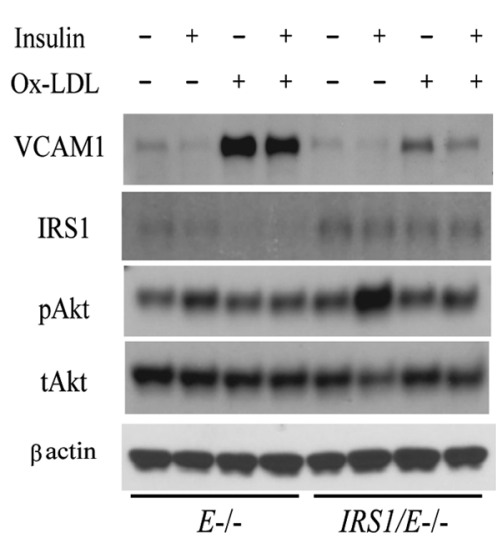

B
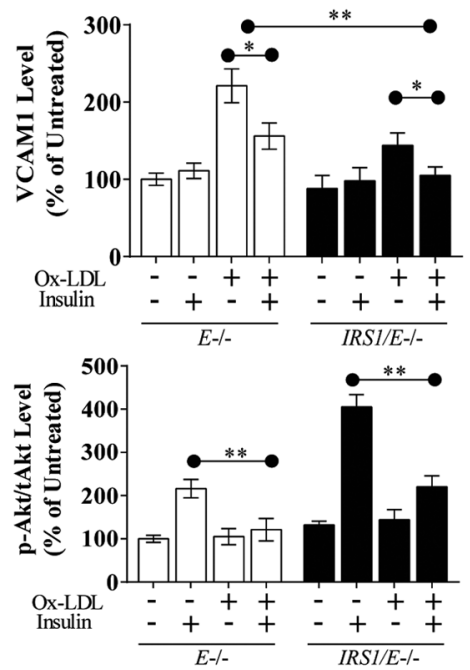

$\mathbf{E}$

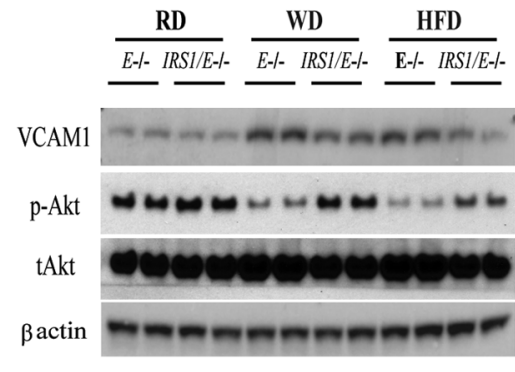

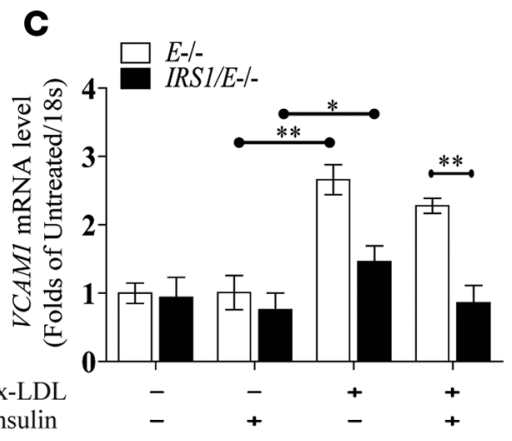

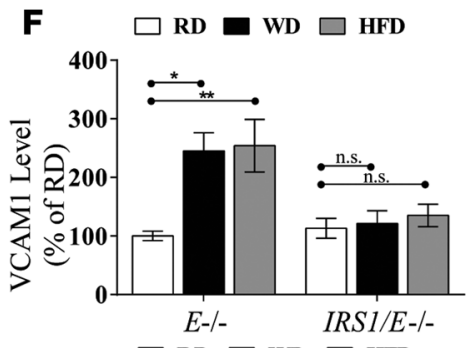

$\square$ RD WD $\square$ HFD

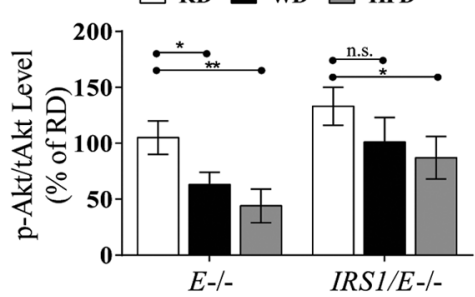

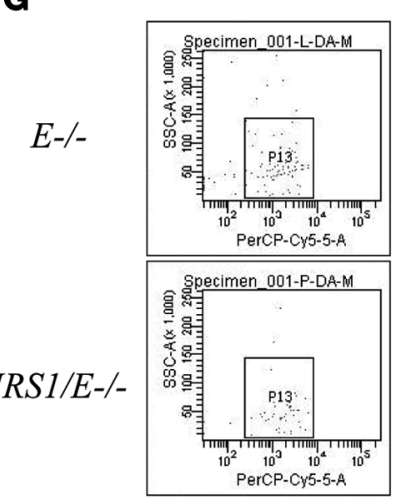

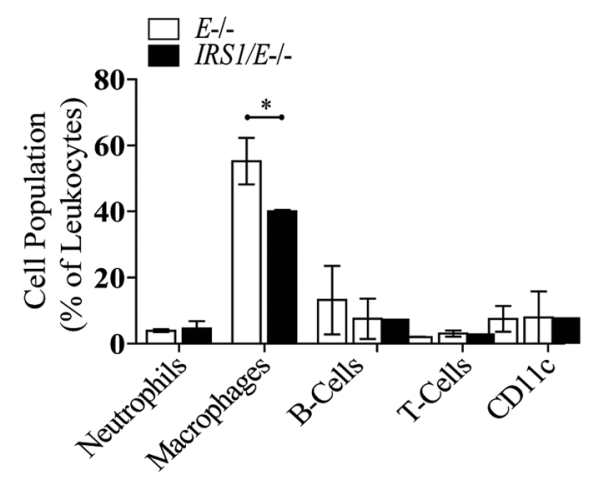

Figure 3. Characterization of VCAM1 expression, leukocyte-endothelial adhesion, and macrophage accumulation in the atherosclerotic lesions. (A and B) IB and densitometry of VCAM1 and p-Akt levels in EC isolated from $E^{-/-}$and IRS1// $E^{-/-}$mice after exposure to ox-LDL for 6 hours in the absence of or presence of insulin treatment. The data are the mean \pm SEM of triplicate samples from 3 separate experiments. Under same condition as (A), (C) VCAM1 mRNA expression was measured in ox-LDL-treated EC. (D) Specific binding of fluorescent-labeled monocytes to EC isolated from $E^{-/-}$and $I R S 1 / E^{-/-}$mice after exposure to ox-LDL for 6 hours. (E and F) IB and densitometry of VCAM1 and p-Akt in aortas from Apoe ${ }^{-/-}$and Irs $1 /$ Apoe $^{-/-}$mice fed WD or HFD for 12 weeks. Results from 3 representative mice are shown for each genotype. (C) Leukocyte levels in whole aortas and thoracic and abdominal aortic lesions of Apoe $^{-/-}(n=6)$ and Irs1/Apoe ${ }^{-/-}$mice $(n=6)$, as assessed by flow cytometry. Data are represented as mean \pm SEM of at least 5 mouse replicates or cellular replicates for VCAM1 expression and leukocyte-endothelial binding experiments. ${ }^{*} P<0.05,{ }^{*} P<0.01$ (2-way/3-way ANOVA for multiple comparisons involving 2/3 factorial variables and 2-tailed Student's $t$ test for pairwise comparisons).

were significantly decreased from $57 \%$ to $39 \%$ in Irs $1 / \mathrm{Apoe}^{-1-}$ mice versus Apoe ${ }^{-1-}$ mice (Figure 3G), without changes in neutrophils or B or T lymphocytes.

Insulin's differential regulation of EDNRB expression in EC. Since p-eNOS was elevated in EC and aortas from Irs $1 / A_{p o e^{-/}}$mice, arterial functional studies were performed. Acetylcholine (Ach) significantly dilated 
A

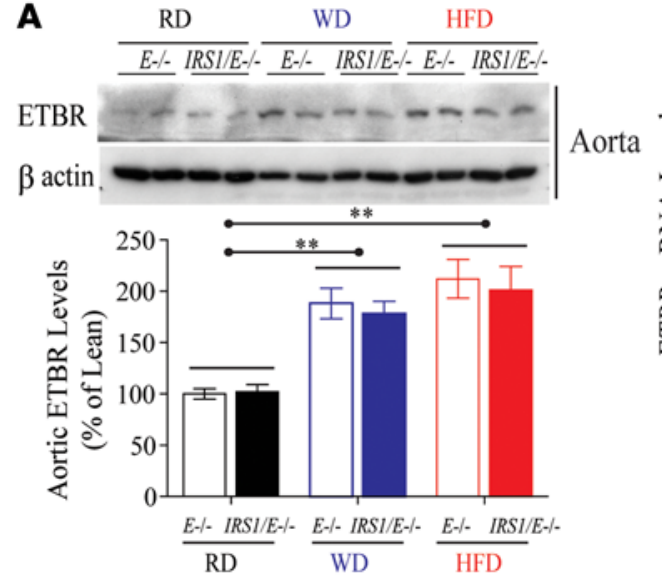

C

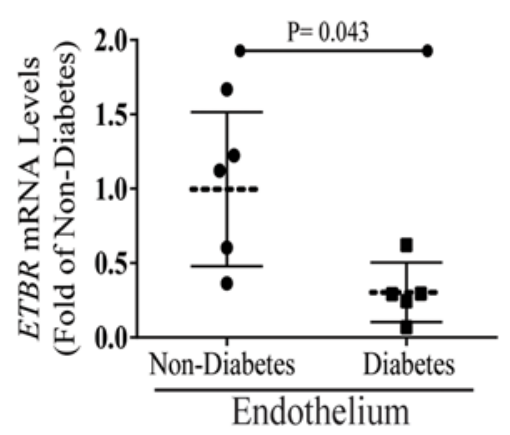

E

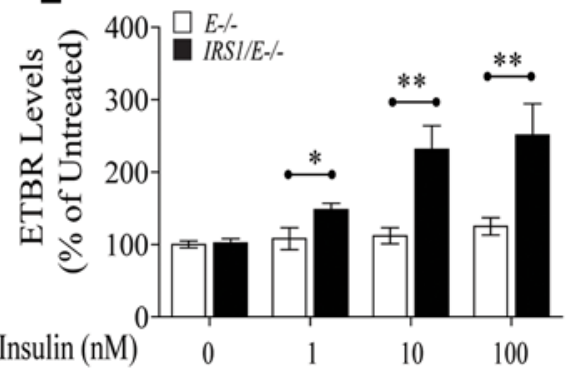

B

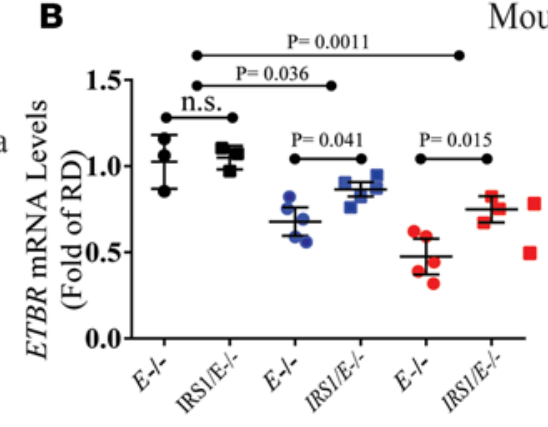

Mouse Aorta

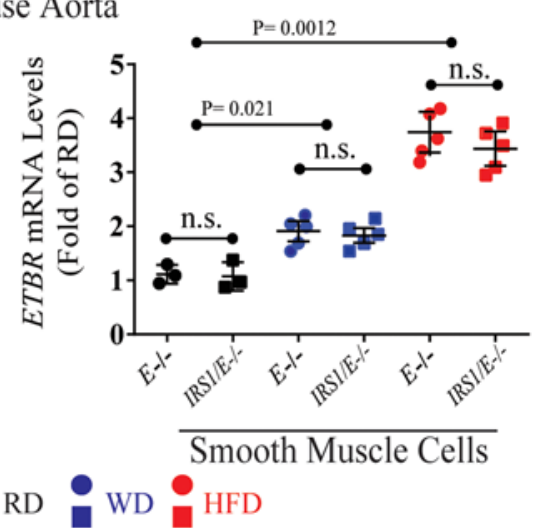

D

Endothelial Cells

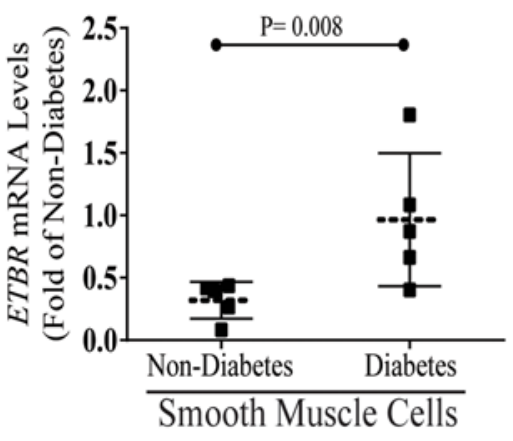

$\mathbf{F}$

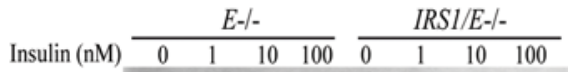

ETBR

$\beta$ actin

IRS1

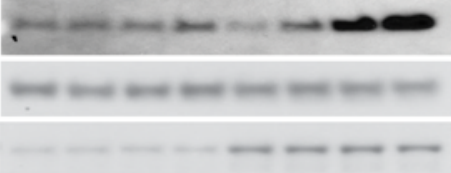

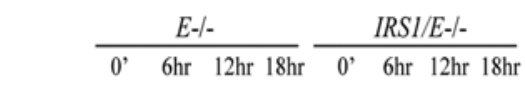

ETBR

$\beta$ actin

IRS1

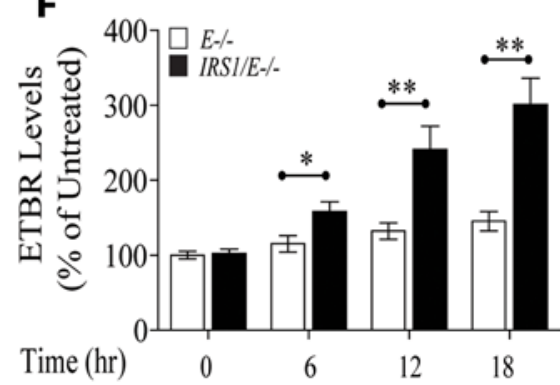

Figure 4. Differential regulation and expression of EDNRB in EC versus VSMC from diabetic patients and rodents. (A) IB of EDNRB in aortas from $A p e^{-/-}$ and Irs1/Apoe $e^{-/-}$mice fed WD or HFD for 12 weeks. (B) EDNRB mRNA expression in aortic endothelia and in VSMC from Irs1/Apoe ${ }^{-/-}$( $n=5$ per group) and $A p o e^{-/-}$mice ( $n=5$ per group) on WD or HFD. Basal (RD) mRNA expression in Apoe $e^{-/-}$mice was set to 1. (C) Quantitative EDNRB mRNA expression in media and intima of human mammary arteries from obese patients with $(n=5)$ or without diabetes $(n=5)$. (D-F) IB and quantification of EDNRB in EC after insulin stimulation with $(\mathbf{E})$ dose- or $(\mathbf{F})$ time-dependent manner. Data are represented as mean \pm SEM of at least 5 mouse replicates or cellular replicates for EDNRB expression experiments. ${ }^{*} P<0.05,{ }^{* *} P<0.01$ (2-way ANOVA for multiple comparisons involving 2 factorial variables and 2-tailed Student's $t$ test for pairwise comparisons).

common carotid arteries more in Irs1/Apoe ${ }^{-1-}$ mice than $A p o e^{-1-}$ mice, but no differences were observed when sodium nitroprusside (SNP) was applied (Supplemental Figure 6). To understand the mechanism that could be responsible for the enhanced responses to Ach, we compared the gene expression profile of aortas obtained from diabetic and nondiabetic Apoe $e^{-/}$mice. Using criteria of $P<0.05$ and 2-fold changes, diabetes increased 76 genes and decreased 56 genes in the aortic regions, compared with nondiabetic mice. Heatmap analysis showed the top 8 upregulated and 19 downregulated genes. One of the genes, EDNRB, was selected since it causes vasodilation in EC and vasocontraction in VSMC (Supplemental Figure 7A and refs. 16, 23-25). We and others have reported that EDN1 expression is regulated by insulin (26) and that its activation is dysregulated in diabetes (27). Consistent with microarray data, expression of EDNRB 
A

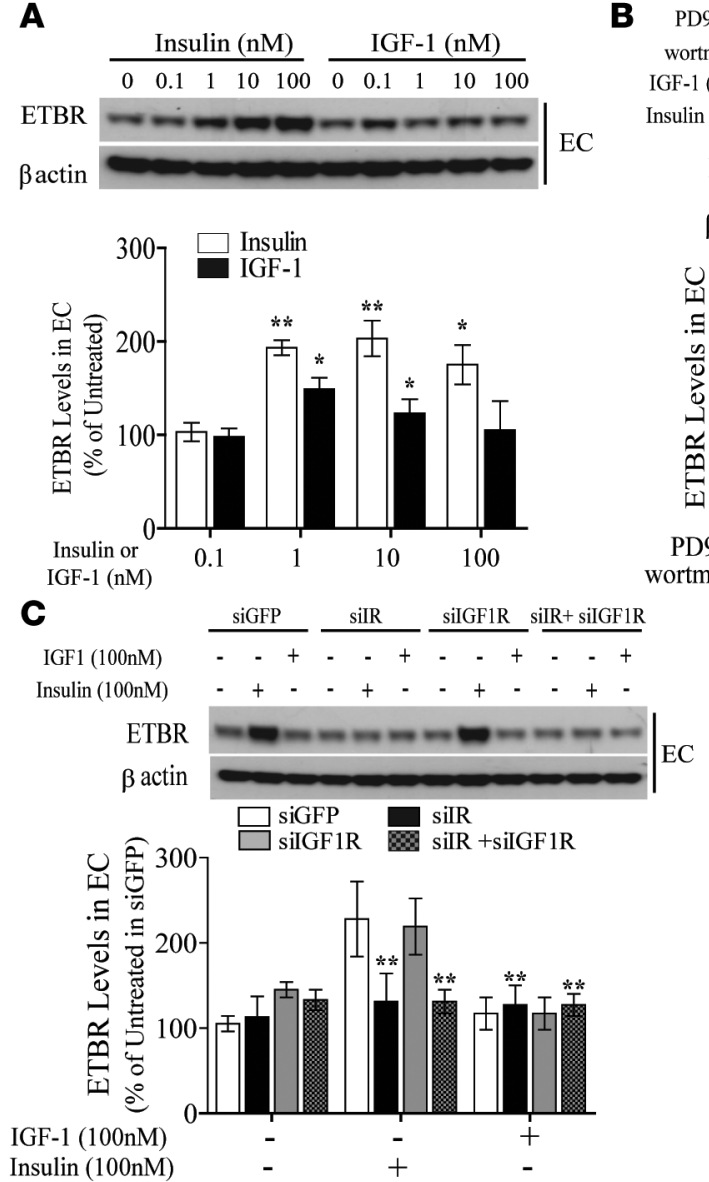

$\mathbf{E}$

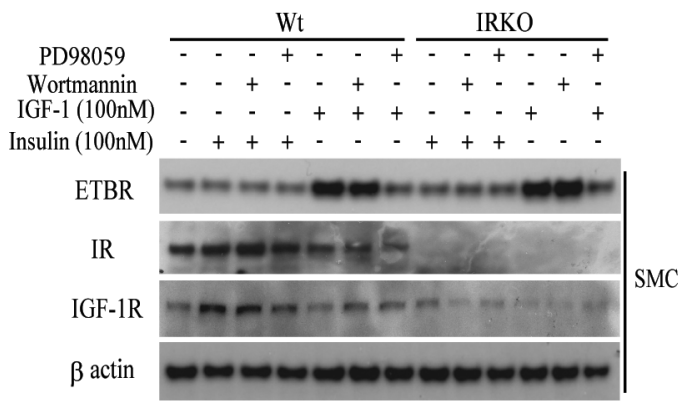

$\begin{array}{ccccccccc}\text { PD98059 } & - & - & - & + & - & - & - & + \\ \text { wortmannin } & - & - & + & - & - & - & + & - \\ \text { IGF-1 }(100 \mathrm{nM}) & - & - & - & - & - & + & + & + \\ \text { Insulin }(100 \mathrm{nM}) & - & + & + & + & - & - & - & -\end{array}$
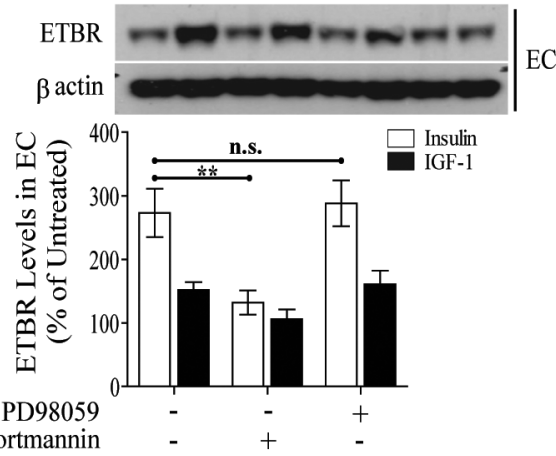

D
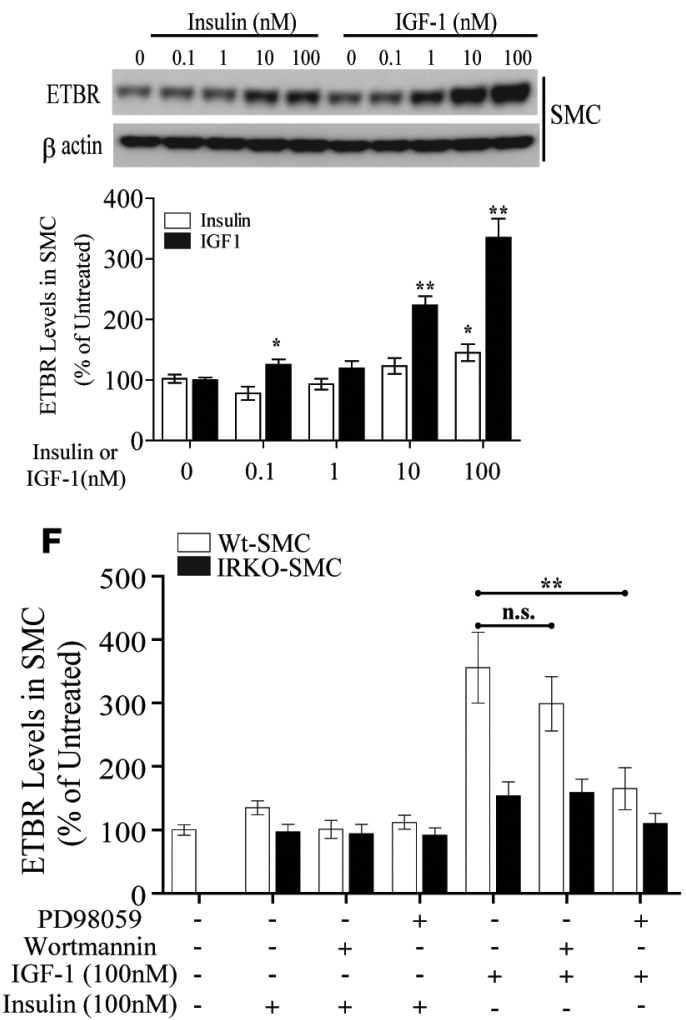

Figure 5. Differential contribution of the PI3K or Erk pathway to EDNRB expression in EC versus VSMC. (A) IB and quantification of EDNRB protein levels

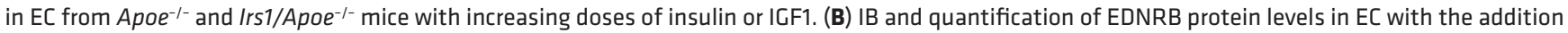
of PI3K inhibitor (wortmannin) and MEK inhibitor (PD98059) either alone or in combination. (C) IB and quantification of EDNRB protein levels in EC following insulin or IGF1 treatment after knockdown of IR, IGF1R, or IR plus IGF1R. (D) IB and quantification of EDNRB protein levels in IGF1-treated VSMC. (E and F) IB of EDNRB protein levels in IGF1-treated VSMC with the combination of wortmannin and PD98059. Data are represented as mean \pm SEM of at least 4 cellular replicates for EDNRB expression experiments in EC or VSMC. ${ }^{*} P<0.05,{ }^{*} P<0.01$ (2-way/3-way ANOVA for multiple comparisons involving $2 / 3$ factorial variables and 2-tailed Student's $t$ test for pairwise comparisons).

mRNA in aortas of $A p o e^{-1-}$ mice fed HFD was increased by 3-fold compared with Apoe $e^{-1-}$ mice fed RD (Supplemental Figure 6, B and C). Further, EDNRB protein levels from whole aortas of Apoe ${ }^{-1}$ and Irs1/ $A p o e^{-1-}$ mice did not differ on RD, but they were elevated by 1.8- and 2.1-fold when Apoe ${ }^{-1-}$ and Irs1/ $A p o e^{-1-}$ mice were fed WD and HFD versus RD, respectively (Figure 4A). When aortic endothelium and media were separated by laser-capture microdissection and studied, EDNRB mRNA expression declined in endothelia by $33 \%$ and $51 \%$ in mice on WD and HFD, respectively, compared with $A p o e^{-/-}$mice on RD (Figure 4B). In contrast, $E D N R B$ mRNA expression in media increased by 1.9- and 3.6-fold, respectively, 
A

DAF-DA

ET-1
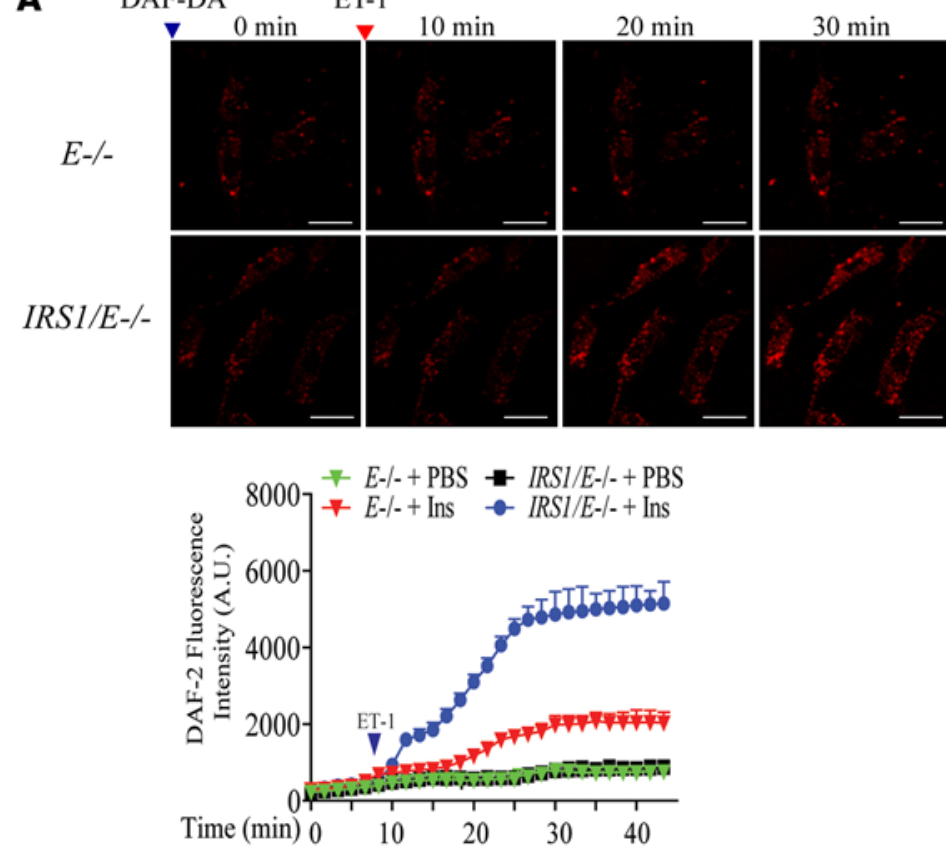

C

$$
\begin{aligned}
& -E-/-+\mathrm{PBS} \quad-I R S I / E-/-+\mathrm{PBS} \\
& -E-/-+ \text { Ins } \quad-I R S I / E-/-+ \text { Ins } \\
& \rightarrow E-/-+ \text { Ins }+ \text { BQ-788 }=\text { IRSI/E-/- + Ins+BQ-788 }
\end{aligned}
$$

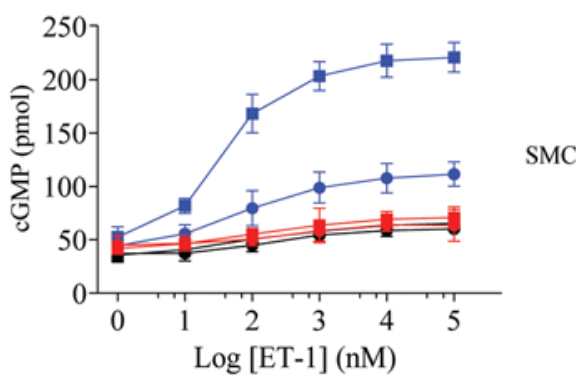

E

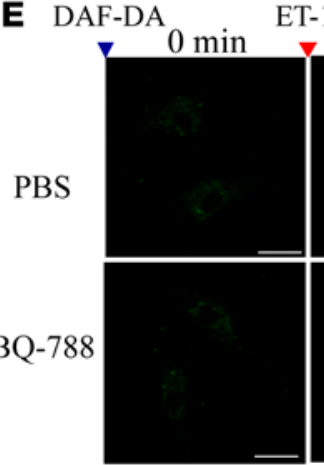

ET-1

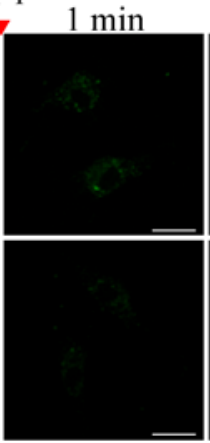

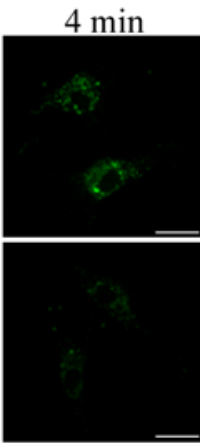

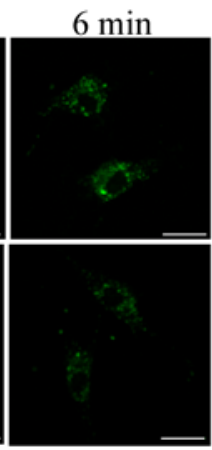

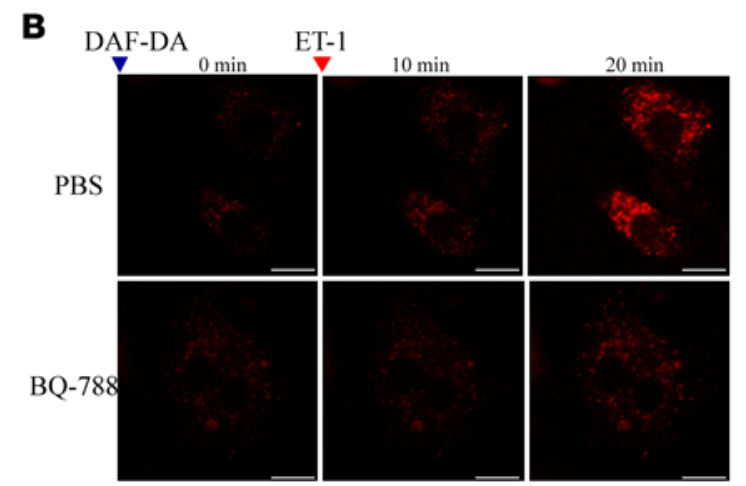

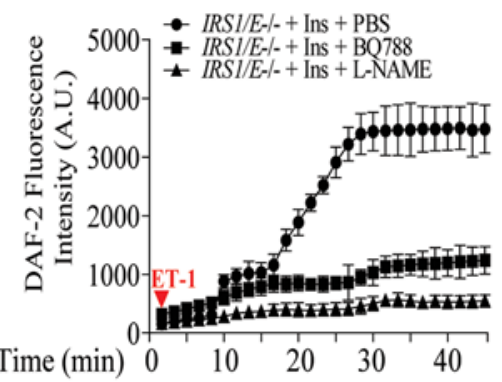

D

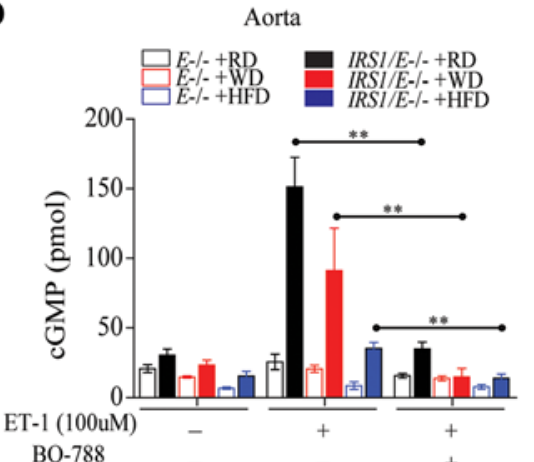

F

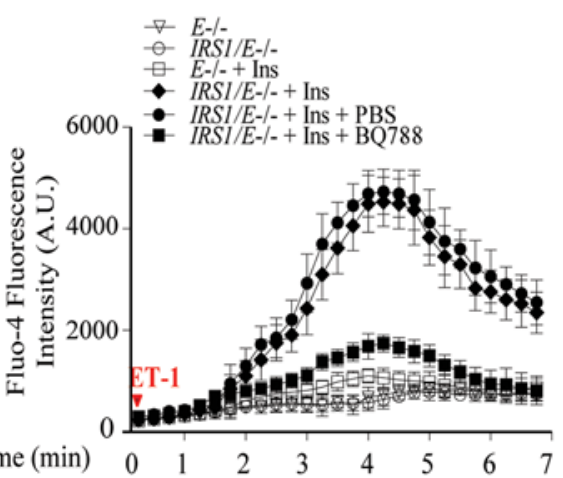

Figure 6. NO production in EC from Irs1/Apoe ${ }^{-/-}$mice and its relationship to EDN1 and EDNRB. (A) EDN1-induced NO production in the aortic EC from Irs1/ $A p o e^{-/-}$and $A p o e^{-/-}$mice. NO production was visualized by DAF-2DA fluorescence intensity (FI) and quantified. Original magnification, $\times 15 ;$ scale bar: $5 \mu$ m. (B) EDN1-induced NO production in insulin-stimulated aortic EC from Apoe ${ }^{-/-}$and Irs1/Apoe $e^{-/-}$mice pretreated with BQ-788 (0.1 mM) or L-NAME (0.5 mM). NO production was visualized by DAF-2DA and quantified. Original magnification, $\times 20$; scale bar: $5 \mu \mathrm{m}$. (C) N $\omega$-nitro-L-arginine-sensitive accumulation of cGMP in VSMC from Apoe ${ }^{-/-}$and Irs1/Apoe ${ }^{-/-}$mice stimulated with EDN1 pretreated with BQ-788 or L-NAME. (D) Accumulation of cGMP in aortas from WD- or HFD-fed $A p o e^{-/-}$and Irs1/Apoe $e^{-/-}$mice. (E and F) Ca $\mathrm{Ca}^{2+}$ accumulation was visualized and quantified by Fluor-4 in EC. Original magnification, $\times 15$; scale scale bar: $5 \mu \mathrm{m}$. Data are represented as mean \pm SEM of at least 5 cellular replicates for NO production experiments in EC and cGMP experiments in VSMC. ${ }^{* *} P<0.01$ (3-way ANOVA for multiple comparisons involving 3 factorial variables and 2-tailed Student's $t$ test for pairwise comparisons). 
A
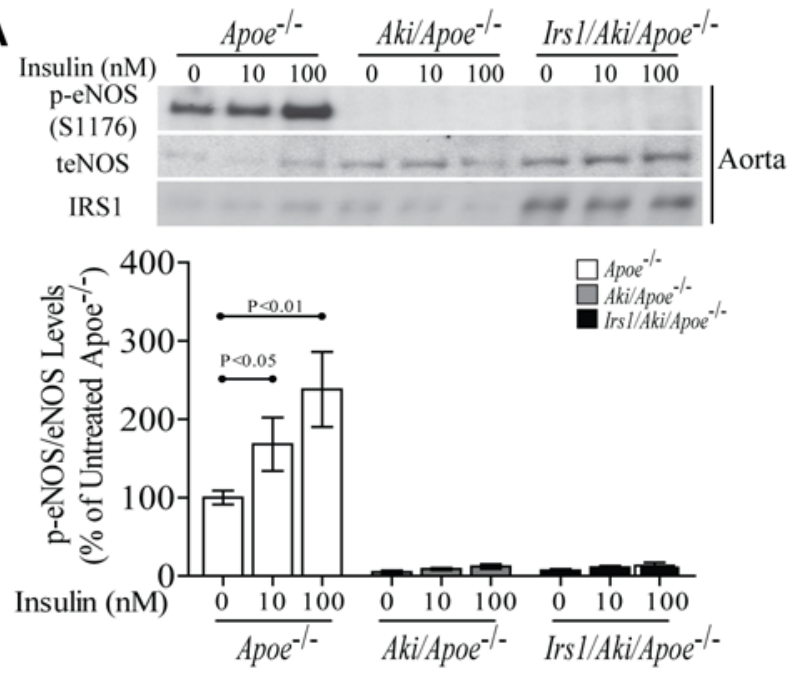

C

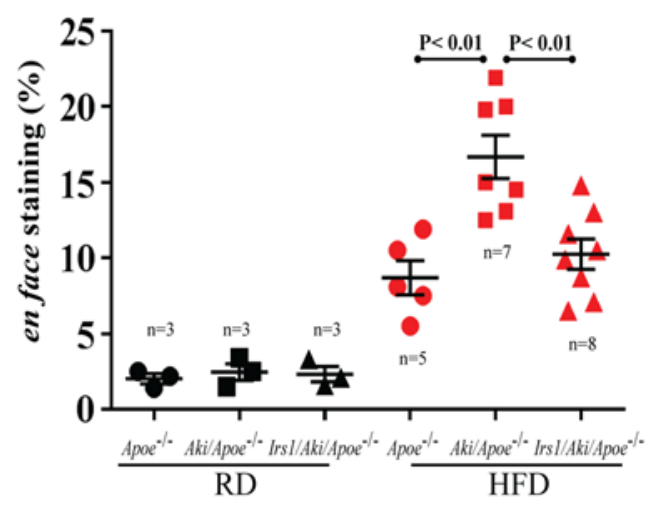

B
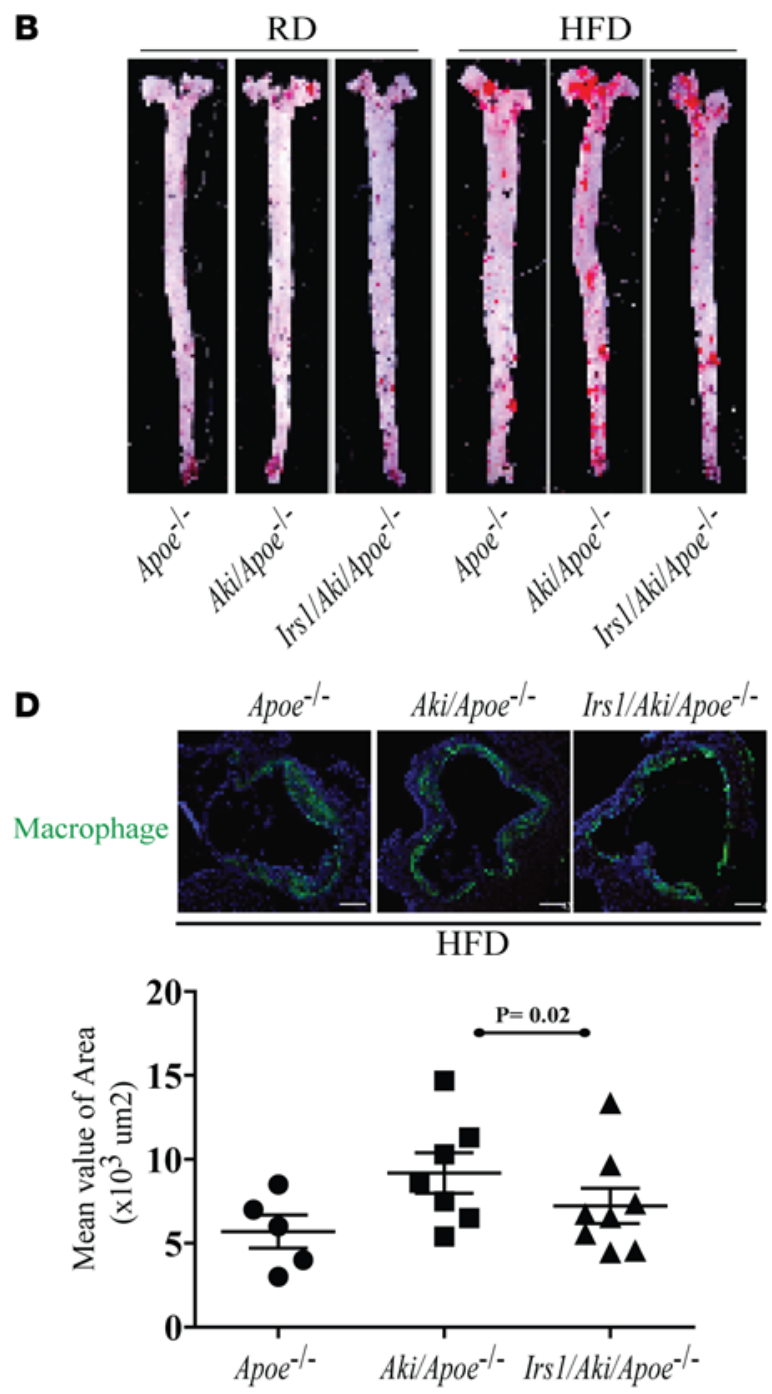

while on WD and HFD. Interestingly, EDNRB mRNA expression in endothelia from Irs $1 /$ Apoe $^{-1}$ mice was increased by $31 \%$ and $75 \%$ compared with that from Apoe $e^{--}$mice when fed WD and HFD, respectively, whereas no differences were observed in the smooth muscle cell (SMC) layer between the two types of mice (Figure 4B). EDNRB mRNA expression was also studied in tunica media and SMC layers in arteries obtained from human subjects (Supplemental Table 1). EDNRB mRNA levels were reduced by $68 \%$ in endothelia and increased by 3.2 -fold in the media of mammary arteries from diabetic versus nondiabetic patients and rodents (Figure 4, B and C). The results showed that the EDNRB expression in EC from Irs1/ $\mathrm{Apoe}^{-1-}$ mice was significantly greater than that in EC from $\mathrm{Apoe}^{-/-}$mice in both insulin dose- and timedependent manner (Figure 4, D-F). To evaluate the mechanism for the differential regulation of EDNRB expression in EC and VSMC, the effect of insulin and IGF1 on the EDNRB expression was studied. Insulin at $1 \mathrm{nM}$ and $100 \mathrm{nM}$ increased EDNRB protein levels in EC from IrsI/Apoe ${ }^{-1-}$ mice by 1.5- to 2.4-fold after 6 hours, whereas no change was observed with IGF1 (Figure 5A). Inhibitors of PI3K (wortmannin) reduced insulin-induced EDNRB expression in EC, but inhibitors of MEK (PD98059) were not effective (Figure 5B). The application of siRNA targeting the insulin receptor (siIR) completely inhibited insulinstimulated EDNRB induction similar to the addition of siIR plus siIGF1R but siIGF1R alone was not 
A

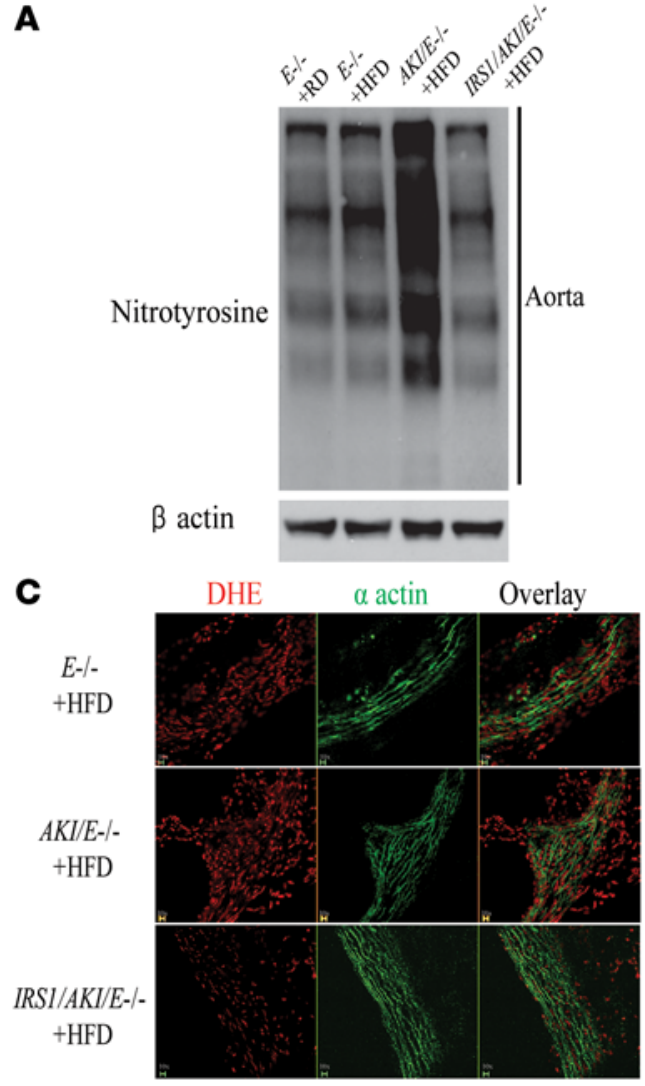

B

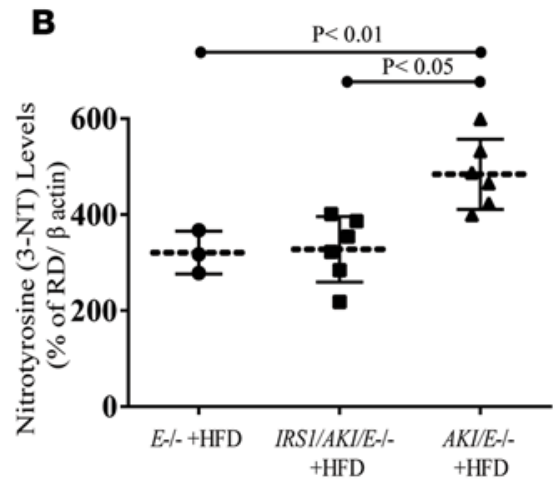

D

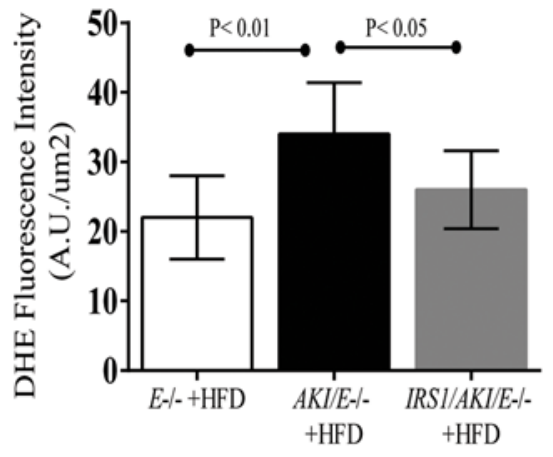

Figure 8. Effect of mutated eNOS (AKI) on HFD-induced oxidative stress in the aorta. (A) IB and (B) densitometry of NT levels in aortas of $A p o e^{-I_{-}}(n=3)$, Aki/Apoe ${ }^{-/-}(n=6)$, and Irs1/Aki/Apoe ${ }^{-/-}$ $(n=6)$ mice fed HFD. (C) Staining and (D) fluorescent density of DHE in aortas under the same conditions as in $\mathbf{A}$. Original magnification, $\times 10$; scale bar: $100 \mu \mathrm{m}$. Results are mean \pm SEM of at least 5 mouse replicates. $P<0.05, P<0.01$ (1-way ANOVA for multiple comparisons involving 1 factorial variable and 2-tailed Student's $t$ test for pairwise comparisons).

effective (Figure 5C). In VSMC, IGF1 at $0.1 \mathrm{nM}$ to $100 \mathrm{nM}$ significantly increased EDNRB expression by 3.3-fold. In contrast, insulin increased EDNRB protein expression by 2.1-fold in VSMC only at $100 \mathrm{nM}$ (Figure 5D). Interestingly, IGF1 increased EDNRB expression in VSMC from Apoe ${ }^{-/-}$mice by 3.6-fold, which was inhibited by PD98059 but not by wortmannin (Figure 5, E and F), demonstrating the preferential regulation of EDNRB in EC by insulin and in VSMC by IGF1.

Mechanisms of insulin's induction of NO production via EDNRB. The mechanism of insulin's effect to increase expression of insulin-induced EDNRB and NO production was studied in EC cultured from Irs1/Apoe $e^{-/-}$and Apoe $e^{-/-}$mice. Without insulin, EDN1 (10 nM) increased NO production (measured by DAF-2) by only $20 \%$ in EC from Irs1/Apoe $e^{-1-}$ and Apoe $e^{-/-}$mice. However, after preincubation with insulin for 16 hours, EDN1 $(10 \mathrm{nM})$ increased NO production dramatically in EC from Irs1/Apoe ${ }^{-/-}$ mice by 5.5-fold and in EC from Apoe ${ }^{-1-}$ mice by 1.6-fold (Figure 6A). Confocal microscopy showed that a selective antagonist of EDNRB, BQ-788 (28), and L-NAME greatly reduced EDN1-induced NO production in EC preincubated with insulin by $77 \%$ and $95 \%$, respectively (Figure $6 \mathrm{~B}$ ). Coculturing of VSMC (bottom) with EC (top) using cells isolated from Irs 1/Apoe $e^{-/-}$and Apoe $e^{-/-}$mice showed that after preincubation with insulin, EDN1 increased intracellular cGMP levels in VSMC by 4.4-fold in Irs1/ Apoe ${ }^{-/-}$mice versus Apoe ${ }^{-/-}$mice; this effect was abolished by BQ-788 (Figure 6C). Basal cGMP levels in aortas from Irs 1/Apoe $\mathrm{A}^{-/-}$mice versus $\mathrm{Apoe}^{-/-}$mice were $26.6 \mathrm{pmol} /$ well versus $20.8 \mathrm{pmol} /$ well on RD and were decreased in both types of mice on WD and HFD. However, with the addition of EDN1 (100 $\mathrm{nM}$ ), cGMP levels in aortas were 6-fold higher in Irs1/Apoe $e^{-/}$mice versus Apoe $e^{-/}$mice on RD. When on WD and HFD, EDN1-induced cGMP levels decreased by 43\% and 71\% versus RD in Irs $1 /$ Apoe $^{-/}$ mice but were still 3.2- or 2.2-fold above those from $A p o e^{-/-}$mice. The addition of BQ-788 abolished EDN1's stimulation of cGMP in aortas from both types of mice (Figure 6D). A maximum $\mathrm{Ca}^{2+}$ accumulation, induced by EDN1 and assessed by fura-4 $(29,30)$, was found in EC from Irs1/Apoe $e^{-1}$ mice in the presence of insulin (4.3-fold above EC from $A p o e^{-1-}$ mice), which was inhibited by $76 \%$ with the addition of BQ-788 (Figure 6, E and F). Insulin's effect on EDNRB expression increased in EC from Irs $1 / A_{p o e^{-/-}}$mice by 3.3-fold above that from Apoe ${ }^{-1-}$ mice, which correlated with increased eNOS and Akt activation, but these effects were inhibited inhibited by wortmannin (Supplemental Figure 8, A and 
A

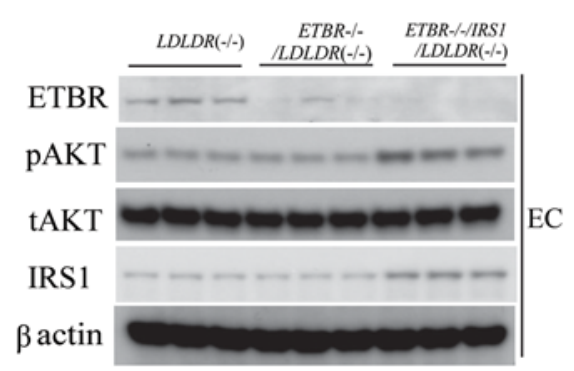

D
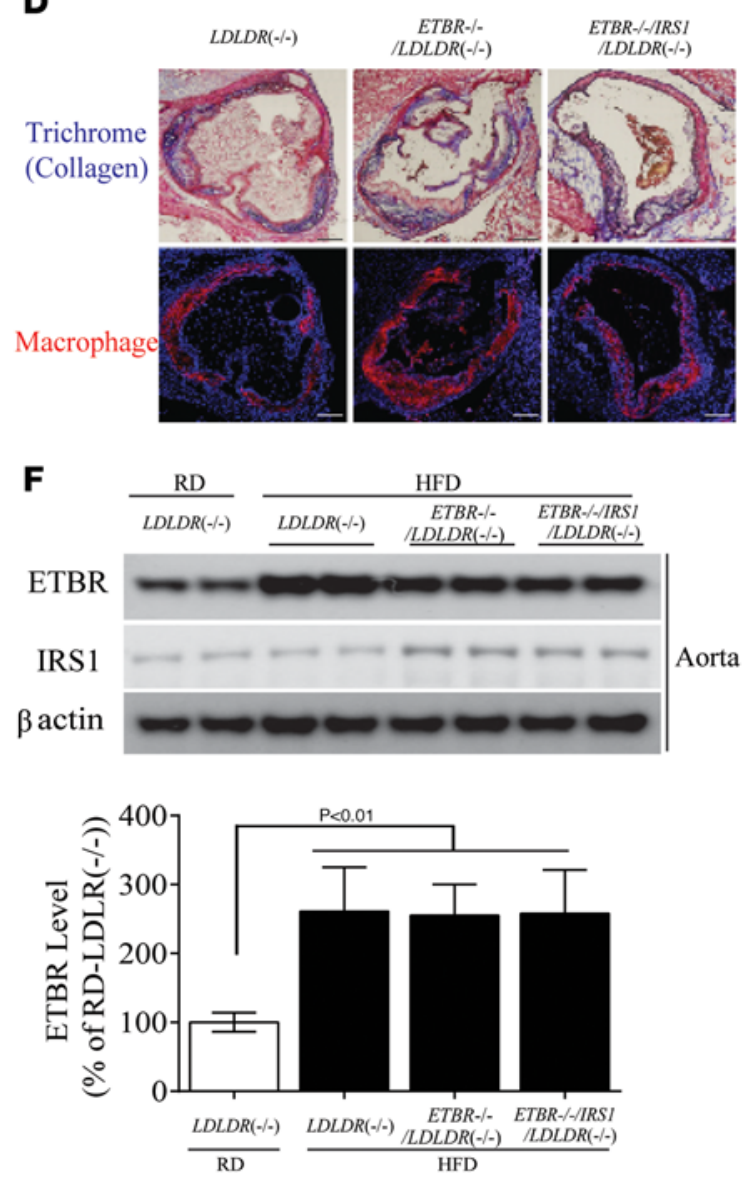

B

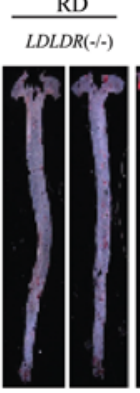

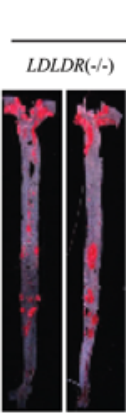

HFD

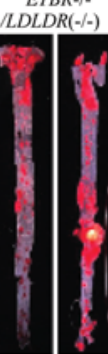

E

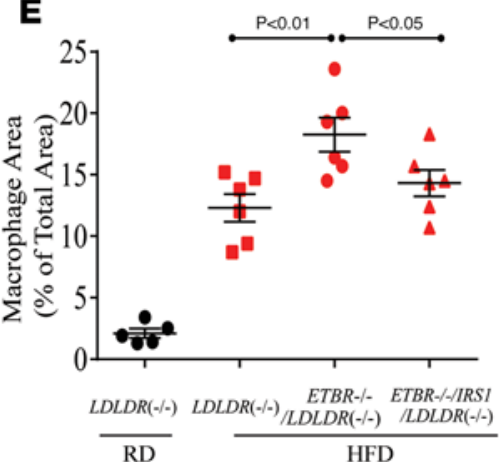

G
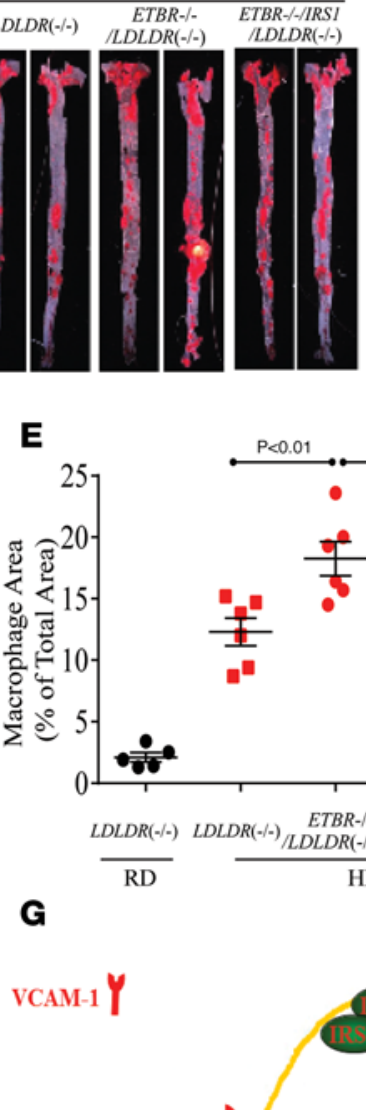

C

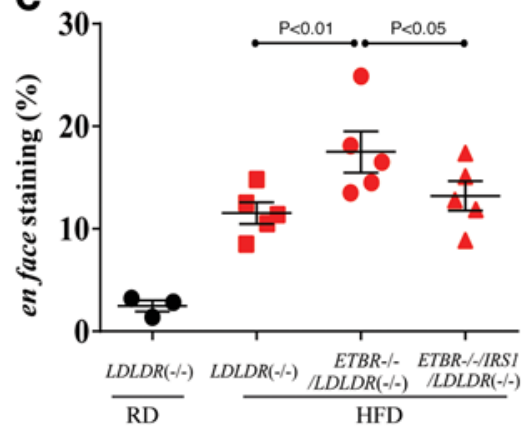

$\mathbf{F}$

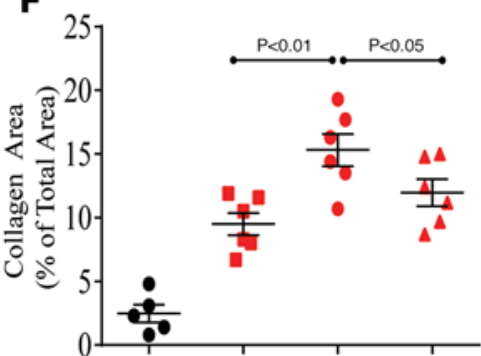
$\operatorname{LDLDR}(-/-) \operatorname{LDLDR}(-/-) \quad E T B R-/=E T B R-/ / I R S I$ $\frac{R}{\text { RD }} \frac{\text { HFD }}{\text { HF }}$

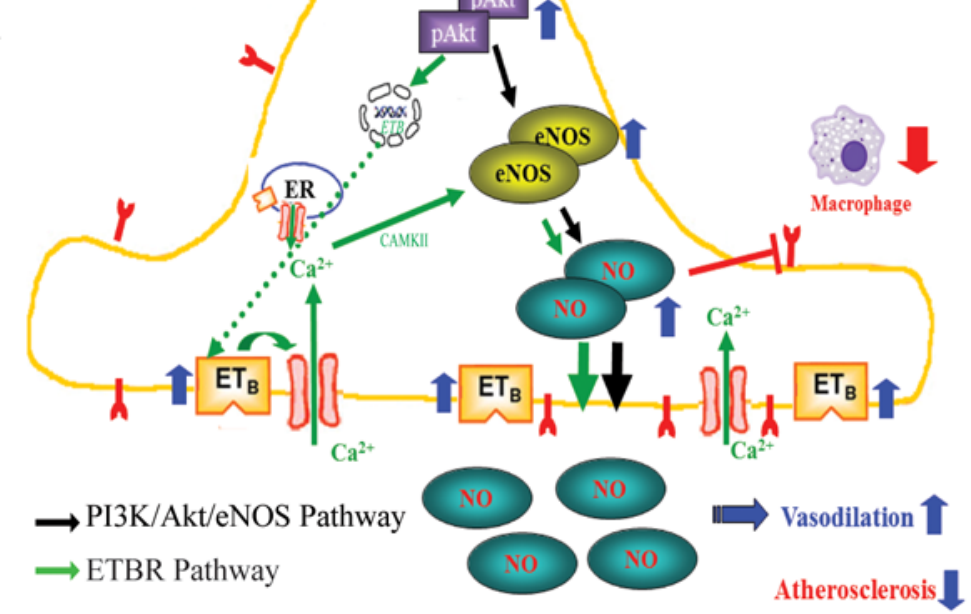

Figure 9. Effect of endothelial EDNRB signaling on atherosclerosis in LdIr-1- mice. (A) IB of EDNRB protein levels in EC from Ldlr $r^{-1-}(n=6)$, Ednr $b^{-1-} / L d l r^{-1-}$ $(n=6)$, and Irs1/Ednrb ${ }^{-1-} / L d l r^{1-}$ mice $(n=6)$. EDNRB and p-Akt expression were separately blotted in different gels. (B) Representative en face staining and

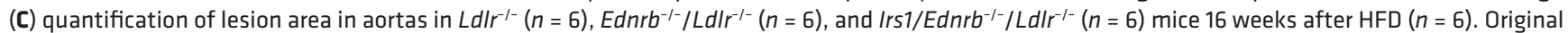
magnification, $\times 2$. (D) Histological analysis and $(E)$ quantification of cross sections from the aortic sinus stained with trichrome and immunostained with anti-MAC2. Original magnification, $\times 4$; scale bar: $100 \mu \mathrm{m}$. (F) IB of EDNRB in aortas from Ldlr $r^{-1-}(n=6), E d n r b^{-1-} / L d l r^{-1-}(n=6)$, and Irs1/Ednrb ${ }^{-1-} / L d l r^{-1-}$ $(n=6)$ mice fed HFD for 16 weeks. (C) Schematic mechanism of increased NO production in EC from Irs $1 / A$ poe ${ }^{-/-}$mice. Data are represented as mean \pm SEM of at least 6 mouse replicates. $P<0.05, P<0.01$ (1-way ANOVA for multiple comparisons involving 1 factorial variable and 2 -tailed Student's $t$ test for pairwise comparisons).

B). It has been reported that the interaction of EDN1 with EDNRB induces activation of CAMKII and enhances NO production (31). When EDNRB expression was reduced by $90 \%$ using siEDNRB plasmid, p-CAMKII was also reduced by $84 \%$ in EC from $I R S 1 / E^{-/-}$mice without altering p-eNOS Ser1176 (Supplemental Figure 8, C and D). The addition of siRNA targeting EDNRB inhibited NO production in EC from Irs $1 / \mathrm{Apoe}^{-/}$mice incubated with insulin and EDN1 by $70 \%$ compared with cells overexpressing siScramble (Supplemental Figure 9, A and B). 
Insulin's effect on NO production and atherosclerosis independent of p-eNOS at Ser1176. To determine specifically whether insulin's enhancement of EDN1-induced NO production was due to p-eNOS at Ser1176 (32), we substituted Ser1176 of eNOS with alanine (AKI), which was overexpressed in EC using adenoviral vectors (adAKI). EC from Apoe ${ }^{-1-}$ mice infected with AdGFP, preincubated with insulin, and stimulated with EDN1 had 1.4-fold increase in NO, similar to EC overexpressing only AKIeNOS (Supplemental Figure 10A). However, EC overexpressing IRS1 with preincubation of insulin and addition of EDN1 had dramatically increased NO production by 9.2-fold. Further, in EC overexpressing AKI and IRS1, preincubation with insulin and addition of EDN1 still increased NO production by 4.1 -fold, a 55\% reduction compared with EC overexpressing IRS1, which is still 2.1-fold above EC (AdGFP) (Supplemental Figure 10A). Similarly, the addition of BQ-788 completely inhibited NO production in EC overexpressing IRS1 with or without AKI expression (Supplemental Figure 10B). IB of AKI-overexpressing EC and aortas of $A k i$ mice showed that insulin increased p-eNOS significantly in IRS1-overexpressing EC and control aortas, but p-eNOS was inhibited in AKI-overexpressing EC and aortas of Aki mice (Supplemental Figure 11A and Figure 7A). In an ex vivo study, EDN1 increased NO production in aortas from Irs1/Apoe ${ }^{-/-}$mice on HFD compared with that of Apoe $e^{-/-}$mice by 2.7-fold, which was inhibited by BQ-788 (Supplemental Figure 11, B and C).

To determine whether activation of Ser1176 NOS-independent pathways can affect the severity of atherosclerosis, we bred Aki mice or Irs1/Aki mice with Apoe $e^{-1}$ mice and derived Aki/Apoe ${ }^{-/}$and Irs1/Aki/ $A p o e^{-1-}$ mice, which were fed HFD for 16 weeks. Metabolically, these mice did not differ in weight, blood glucose, fasting insulin, or total cholesterol and triglyceride levels. Consistent with the impaired activation of Ser1176 NOS in infected EC with adAKI, insulin-induced p-eNOS (Ser1176) was blunted in aortas from both Aki/Apoe $e^{--}$and Irs1/Aki/Apoe ${ }^{-/-}$mice (Figure 7A). On RD or HFD, analysis by en face assessment showed that $A k i / A p o e^{-/-}$mice had greater severity of atherosclerosis than $A p o e^{-1-}$ mice by $47 \%$, but atherosclerosis was decreased in Irs1/Aki/Apoe ${ }^{-1-}$ mice (Figure 7, B and C). The extent of lipid deposition in Irs1/ Aki/Apoe $e^{-1-}$ mice was 35\% less than in Aki/Apoe $e^{-1}$ mice and similar to that in Apoe ${ }^{-/-}$mice. Inflammation in the aortic wall was assessed by galectin-3 (macrophage) content, which increased by $27 \%$ compared $A K I / E^{-/-}$versus $E^{-/-}$mice, which was reduced by $19 \%$ in Irs $1 / A k i / A p o e^{-/-}$mice (Figure 7D). To determine whether the lowering of oxidative stress in Irs $1 / \mathrm{Apoe}^{-/-}$mice was due to uncoupling eNOS activity independent of p-eNOS at Ser1176, NT levels and oxidative stress were also studied in Aki/Apoe ${ }^{-1-}$ mice and Irs1/ $A k i / A p o e^{-1-}$ mice. The levels of NT and oxidative stress in aortas from $A k i / A p o e^{-/-}$mice were increased by $41 \% \pm 14 \%$ and $62 \% \pm 21 \%$, respectively, compared with $\mathrm{E}^{-/-}$mice, whereas aortas from Irs $1 /$ Aki $^{\prime}$ Apoe ${ }^{-/-}$ mice exhibited significantly lower $(36 \% \pm 16 \%)$ NT levels (Figure $8, \mathrm{~A}$ and $\mathrm{B})$ and DHE staining $(31 \% \pm$ $12 \%$ ) than those from Aki/Apoe ${ }^{-1-}$ mice (Figure 8, C and D).

Effect of the endothelial EDNRB pathway on atherosclerosis. To determine directly the role of the endothelial EDNRB pathway on atherosclerosis in a separate model of atherosclerosis, we used CDH5-CREmediated recombination to generate mice lacking EDNRB in vascular EC of $\mathrm{Ldlr}^{-{ }^{-}}$mice. EDNRB protein levels were undetectable by IB analysis in the EC of both control Ednrb ${ }^{-/-} / L d l r^{--}$and Irs $1 / E d n r b^{-1-} /$ $L d l r^{-1}$ mice (Figure 9A). Sixteen weeks of HFD resulted in hyperglycemia, hyperinsulinemia, and dyslipidemia in $\mathrm{Ldlr}^{-1}$ mice, whereas these mice had similar glucose tolerance, insulin sensitivity (Supplemental Figure 12, A and B), BW (data not shown), plasma insulin, and total cholesterol and triglyceride levels (Supplemental Figure 12, C and D). These data indicate that the lack of the EDNRB pathway in EC doesn't affect systemic insulin sensitivity. The effect of endothelial-specific EDNRB deficiency on the severity of atherosclerosis was characterized by multiple methods. Sudan IV en face staining of dissected aortas from $E d n r b^{-1-} / L d l r^{-1}$ mice showed a 32\% increase in the atherosclerotic area, compared with HFD-fed $\mathrm{Ldlr}^{-1}$ mice (Figure 9, B and C). In addition, there was a significant increase in macrophage and collagen content in the aortic root of $\mathrm{Ednrb}^{-1-} / \mathrm{Ldlr^{-1 }}$ mice by $35 \%$ and $29 \%$ (Figure 9, D and E) versus $L d l r^{-1-}$ mice. In contrast, atherosclerotic areas were decreased by $21 \%$ in $I r s 1 / E d n r b^{-1-} / L d l^{\prime-1}$

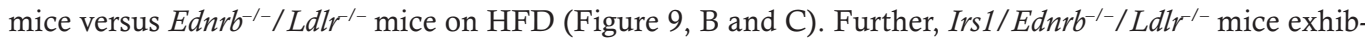
ited fewer macrophages and less collagen content in lesion area, compared with $E d n r b^{-1-} / L d l r^{-1}$ mice by $22 \%$ and $19 \%$, respectively (Figure $9, \mathrm{D}$ and $\mathrm{E}$ ), indicating that phosphorylation of the PI3K/Akt/ eNOS pathway still has antiatherogenic action in $I r s 1 / E d n r b^{-1-} / L d l r^{1-}$ mice, which lack EDNRB in EC. EDNRB protein expression levels in vivo in whole aortas from Irs $1 / E d n r b^{-1-} / \mathrm{Ldll}^{-1-}$ mice were not different, compared with $L d l r^{--}$and $E d n r b^{-1-} / L d l r^{--}$mice with HFD, but EDNRB expression was elevated by 4.2 -fold in these three groups of mice on HFD when compared with those mice on RD (Figure 9F). 
Consequently, the increase in the atherosclerosis correlated with ablation of EDNRB signaling in endo-

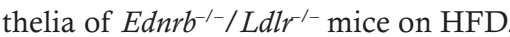

\section{Discussion}

The present study provides conclusive evidence that enhancing the IRS1/Akt/eNOS pathway selectively in the endothelium improves endothelial dysfunction and decreases atherosclerosis in normal, insulin-resistant, and diabetic states, even in the presence of hyperinsulinemia. Further, we have identified a mechanism by which insulin acts via the IRS1/PI3K/Akt pathway to enhance EDNRB expression and NO production in the EC to decrease atherosclerosis (Figure 9G).

Previous studies could not address the question of whether enhancing insulin signaling could decrease atherosclerosis, since they used primarily mice with insulin signaling inhibited in the endothelium by deleting IR (EIRAKO) (4), Akt ( $\left.A K T 1^{-1-} / A P O E^{-/-}\right)(6)$, and eNOS $(12,33)$. Studies using mice with deletion of IRS1 or IRS2 in EC reported increased intimal hyperplasia in an endothelial injury model, but their effects on the extent of atherosclerosis were not evaluated $(34,35)$. The mechanisms for decreasing atherosclerosis by IRS1 overexpression were multiple, including a decrease in VCAM1 expression and a significant increase in the activation of eNOS. The most interesting discovery was that insulin can enhance EDNRB expression selectively in the endothelium via p-Akt, but not in VSMC, to activate eNOS. This action of insulin on EDNRB expression in the endothelium was detected when insulin's actions were significantly enhanced in EC with IRS1 overexpression. Further, insulin's induction of EDNRB expression is mediated through IRS1 and Akt, not the MAPK pathway, in endothelium.

The result of elevated EDNRB expression in EC by insulin was a dramatically increased activation of eNOS, with several fold elevated NO production. Previously, the only known pathway for insulin to activate eNOS was by Akt-mediated phosphorylation of eNOS at $\operatorname{Ser1176}(8,9)$, which does not require $\mathrm{Ca}^{2+}$ and only increases NO production by less than $50 \%$. However, this study demonstrated that insulin may have a new pathway through EDNRB upregulation to increase NO production in EC independent of p-eNOS Ser1176. Consistent with EDNRB's signaling, this action of insulin on NO production is associated with increases in $\mathrm{Ca}^{2+}$ flux and calmodulin activation, which has not been shown to be involved to activate eNOS by insulin $(36,37)$. This mechanism of insulin to increase NO production via EDNRB was clearly established using a series of studies involving Aki mice and deletion and inhibitors of EDNRB actions and Ednrb ${ }^{-1-}$ $L d l r^{-1}$ mice. Since EC is the main site of EDN1 production on the vascular wall, the autocrine actions of EDN1 can be significant, if IR action on EC can be enhanced in insulin-resistant and diabetic states (38). Interestingly, BP, assessed by tail vein method, did not change in Irs $1 / A_{p o e^{-/}}$mice on RD or HFD, even with hyperinsulinemia and increased NO production. It is possible that small reductions in BP may not have been evident using the tail-cuff method in the mice or that downstream NO actions could be inhibited insulin resistance or diabetic status. Further studies are needed to evaluate BP by femoral artery and venous catheterization system (39). These studies also indicated that there is a paradoxical finding of elevated EDNRB expression in VSMC but a reduction of EDNRB in the endothelium in the presence of diabetes. The paradoxical changes in EDNRB expression are likely to have pathophysiological importance for atherosclerosis, since comparable findings were observed in both diabetic patients with atherosclerosis and HFD-fed Apoe $e^{-1}$ mice. The mechanism of paradoxical change in EDNRB is attributable to the differential effect of insulin and IGF1 on EC, since insulin regulates EDNRB in EC via Akt, as opposed to IGF1, which signals via MAPK activation in VSMC (Figure 5, E and F). Since elevation of IGF1 levels is commonly (40) associated with obesity and diabetes $(41,42)$, increased EDNRB expression in VSMC is likely to occur via the MAPK pathway (43). However, the findings of decreased EDNRB expression in EC with diabetes are most likely related to the selective loss of insulin action in the IRS1/Akt pathway of EC. Finally, the results of EDNRBspecific deletion in EC clearly demonstrated the idea that EDN1 may have severe antiatherosclerotic function, which is in contrast with EDN1 and EDNRB's well-known proatherogenic action in VSMC. Thus, in insulin resistance and diabetes, the excessive risk for atherosclerosis could be partially the result of selective loss of insulin's signaling via the IRS1/PI3K/Akt pathway to enhance EDNRB expression in the EC.

\section{Methods}

Generation of Irs1/ApoE $E^{-/}$, Irs1/Aki/Apoe $e^{-/}$, and Irs1/Ednrb-/-/Ldlr/- mice. For the generation of Irs1/ApoE $E^{-/-}$ mice, the human IRSI CDNA, driven by the VE-cadherin promoter was cloned into the pBluscript II KS (+) vector and bred into the $A p o e^{-/-}$background. Founders were screened by genotyping with PCR using a for- 
ward primer specific to the promoter (5'-ATCTGCAGGCAGCTCACAAAG-3') and a reverse primer for exon 1 of the IRS1 cDNA (5'-CGAAGAAGCGTTTGTGCATGC-3') and second primer set for exon 3 to 5 of IRS1 cDNA (5'-GGAGTGCACCCCTGAACCG-3' and 5'-AGTTTGTCCAATTATGTCACACC). The PCR conditions were $94^{\circ} \mathrm{C}$ for 3 minutes, followed by 35 cycles of $94^{\circ} \mathrm{C}$ for 30 seconds, $54^{\circ} \mathrm{C}$ for 30 seconds, and $72^{\circ} \mathrm{C}$ for 90 seconds. The Irs $1 / A k i / A p o e^{-/-}$mice were created by outbreeding Irs $1 / A p o E^{-1-}$ mice with $A k i / A p o e^{-/-}$mice, which were gifts from Paul L. Huang. Irs1/Aki/Apoe-- mice were screened by PCR. We also generated endothelial-specific Ednrb KO mice by mating floxed Ednrb mice (009063, Jackson Laboratories) with Cdh5-Cre mice (017968, Jackson Laboratories) and Ldlr ${ }^{-1}$ mice (002246, Jackson

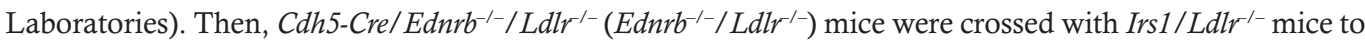
generate Cdh5-Cre/Irs1/Ednrb ${ }^{-/-} / L d l r^{-1}\left(\right.$ Irs1/Ednrb $\left.b^{-1-} / L d l r^{--}\right)$mice.

Chemicals and antibodies. Antibodies used for IB are as follows: t-IRS1 (catalog 2390), Insulin Receptor $\beta$ (IR $\beta$ ) (catalog 3025), IGF1R (catalog 3027), p-CaMKII (catalog 3356), t-CaMKII (catalog 4436), p-Erk (catalog 9101), t-Erk (catalog 4695), p-Akt (catalog 4060), t-Akt (catalog 9272), and NT (catalog 9691) were purchased from Cell Signaling Technology. Anti- $\beta$-actin (sc-1616), goat anti-mouse (sc-2031), and anti-rabbit IgG (sc-2004) were purchased from Santa Cruz Biotechnology. Anti-p-Tyr in IRS1 (Tyr608) (catalog 09-432) was obtained from Sigma-Aldrich and antibodies for IRS1 (catalog 09-248) and Galectin-3 (MABT51) were purchased from Millipore. Anti-VCAM1 (AF643) was obtained from R\&D Systems. Antibodies for p-eNOS (catalog 612392) and t-eNOS (catalog 610297) were purchased from BD Biosciences. Endothelin Receptor type B Ab (NBP1-30599) was obtained from Novus Biologicals. Anti- $\alpha$-actin (catalog A2547), L-NAME (N5751), EDN1 (E7764), wortmannin (W3144), PD98059 (P215), and BQ-788 (B157) were purchased from Sigma-Aldrich. Endothelin-1 ELISA kit (ADI-900-020A) was purchased from Enzo life sciences, and cGMP Direct Biotrak EIA (catalog 45-001-771) was purchased from GE Healthcare Life Sciences. DAF-2DA (cata$\log 251505$ ) was purchased from EMD Millipore Chemicals. Fluo-4 NW Calcium Assay Kit (F36206) was purchased from (Invitrogen). Human Ox-LDL (BT-910) was purchased from Alfa Aesar.

Immunofluorescence and IHC. Serial sections $(10 \mu \mathrm{m})$ were used for trichrome blue staining, and Galectin-3 and $\alpha$-smooth muscle actin were used for immunofluorescence. Stained slides were imaged by using an Olympus FSX100 Microscope (Olympus America).

Assessment of vascular reactivity. Assessment of vascular reactivity was performed in a pressure myograph system (Danish Myo Technologies) as previously described (44) with the following modifications. Briefly, the left carotid arteries were isolated from HFD-fed $\mathrm{Apoe}^{-/-}$and Irs1/Apoe ${ }^{-/-}$mice and mounted onto glass cannulas and maintained at $85 \mathrm{mmHg}$ pressure at $37^{\circ} \mathrm{C}$ in physiologic saline-HEPES buffer oxygenated with $95 \% \mathrm{O}_{2}$ and $5 \% \mathrm{CO}_{2}$. Vessels were constricted with $10^{-5} \mathrm{M}$ phenylephrine followed by stepwise addition of Ach $(0.1 \mathrm{nM}$ to $1 \mu \mathrm{M})$ to generate vasodilator response curves. After basal NO production was blocked by applying $N \omega$-nitro-L-arginine methyl ester (L-NAME) $\left(3 \times 10^{-4} \mathrm{M}, 30\right.$ minutes), vasodilator response curves were generated by stepwise addition of SNP. Acute vasodilator actions of Ach were determined by using cumulative concentration response curves to Ach $(0.1 \mathrm{nM}$ to $1 \mu \mathrm{M})$ in $10^{-5} \mathrm{M}$ phenylephrine-precontracted carotid arteries.

Laser-capture microdissection. Ten sections (10- $\mu \mathrm{m}$ thick) of the each human mammary artery and mouse aorta blocks were cut in a cryostat and placed on slides by the Joslin histology core facility under Rnasefree conditions. The intima and media of the tissue were microdissected from the slide within $30 \mathrm{~min}$ utes of fixation using Arcturus laser-capture microdissection caps and microscope (Invitrogen). The cap was placed on a tube that contained digestion buffer. The tube was inverted until the buffer covered the cap, vortexed, and incubated at room temperature for 5 minutes. The tube was then centrifuged, and the samples were harvested. RNA was isolated according to the RNeasy micro kit protocol (Qiagen) with the following modifications. cDNA was synthesized using $10 \mathrm{ng}$ of extracted total RNA in each sample using the TaqMan MicroRNA Reverse Transcription kit (Applied Biosystems). The human EDNRB and Ednra (hEDNRB-F, GGTTGTGTCCTGCCTTGTGTT; hEDNRB-R, TTCGCATGCACTTGTTCTTGT; hEDNRA-F, GTCCTTTATCCTGGCCATTCC; hEDNRA-R, AGGTTTTATGCTGTTCACCCCTAT) and mouse $E D N R B$ and EDNRA (mEDNRB-F, CAGTCTTCTGCCTGGTCCTC; mEDNRB-R, GGACTGCTTTTCCTCAAACG; mEDNRA-F, GTCCTTTATCCTGGCCATTCC; mEDNRA-R, AGGTTTTATGCTGTTCACCCCTAT) mRNA level were normalized to that of the endogenous control, 18s, and expressed as fold of nondiabetic control. Data were analyzed according to the comparative Ct method (45).

Measurement of $\mathrm{NO}$ and intracellular $\mathrm{Ca}^{2+}$ concentration. EC from $\mathrm{Apoe}^{-/-}$mice and Irs $1 / \mathrm{Apoe}^{-/-}$mice were plated onto gelatin-coated glass coverslips. The NO production and intracellular $\mathrm{Ca}^{2+}$ concentration were 
measured using the membrane-permeable fluorescent marker DAF-2 diacetate $(1 \mu \mathrm{M})$ and Fluo-4 AM (1 $\mu \mathrm{M}$ ), respectively. A ZEISS confocal microscope (LSM 710 NLO) and ZEISS $\times 25$ objective (Plan apochromat, $0.8 \mathrm{NA}$ ) were used for image acquisition. An argon laser $(488 \mathrm{~nm})$ was used for excitation, and the emission band was used for both $\mathrm{NO}$ and $\mathrm{Ca}^{2+}$ imaging at 510 to $550 \mathrm{~nm}$. To minimize photobleaching, all images were collected using $512 \times 512$ scan format at 1 -minute intervals. EC in the absence or presence of the insulin pretreatment for 16 hours were incubated with the fluorescent marker for 30 minutes before image acquisition. On average, 3 to 5 cells were identified in each field of view, and the focal plane was constantly maintained during image acquisition. Adobe Photoshop CS4 software was used for image analysis. The changes in the fluorescence intensity of each cell over time were quantified. Figure 6 and Supplemental Figures 9 and 11 show the representative measurements of $\mathrm{NO}$ and $\left[\mathrm{Ca}^{2+}\right]_{\mathrm{i}}$ (the average of 12 cells), respectively. The changes in Fluo- 4 intensity directly correlate with the changes in $\left[\mathrm{Ca}^{2+}\right]_{i}$.

Flow cytometry analysis. Single aorta cells were prepared using methods described below with modification. The isolated aorta was dissected and cut into small pieces. Aorta pieces were digested with $125 \mathrm{U} / \mathrm{ml}$ collagenase type XI, $60 \mathrm{U} / \mathrm{ml}$ hyaluronidase type I-s, $60 \mathrm{U} / \mathrm{ml}$ DNase I, and $450 \mathrm{U} / \mathrm{ml}$ collagenase type I purchased from Sigma-Aldrich in PBS containing $20 \mathrm{mM} \mathrm{HEPES}$ at $37^{\circ} \mathrm{C}$ for 1 hour. Cells were incubated with propidium iodide (catalog 5135, R\&D System) and stained with CD45-Pacific Blue (catalog 57-0451) and CD14 APC at 1:1,000 (catalog 17-0141) (purchased from Bioscience) and CD19 Alex Fluor 488 at 1: 1,000 (catalog 302219), TCR PerCP/Cys5.5 at 1:1,000 (catalog 109227), CD11c PE/Cy7 at 1:1,000 (cata$\log 117317$ ), and CD68 PE at 1:1,000 (catalog 12-0681) (purchased from BioLegend). Data analysis was performed using FlowJo software.

Microarray analysis. Total RNA was isolated using Trizol (Sigma-Aldrich) from the aorta of each mouse, followed by RNeasy columns with DNase I treatment (QIAGEN). cRNA was generated using standard protocols (Expression Analysis Technical Manual, 2001, Affymetrix) from total RNA and then hybridized to the Affymetrix Mouse Genome 4302.0 Array. Intensity values were quantitated by using MAs5.0 software (Affymetrix). All arrays were normalized for overall intensity using linear regression. Each array was normalized against the microarray most similar to all others by using the central $95 \%$ of expression values. Gene expression analysis was performed using dChip Software (http://www.hsph.harvard.edu/ cli/complab/dchip/). Microarray data were deposited at the Gene Expression Omnibus (National Center for Biotechnology Information) with accession number GSE79623.

Statistics. Values are expressed as mean \pm SEM for continuous variables and as proportions for categorical variables (\%). ANOVA was used to evaluate the main effects and interactions of factor variables on continuous outcomes, and 2-tailed Student's $t$ test was used to compare continuous variables between 2 groups (Supplemental Table 2). $\chi^{2}$ test was used for comparisons of categorical variables. $P$ values of less than or equal to 0.05 were considered statistically significant. STATA SE from StataCorp LP (College Station, Texas, USA) was used to perform all analyses.

Study approval. Use of discarded arterial tissue from patients undergoing coronary artery bypass surgery was reviewed and approved by the regional ethical committee in Odense University Hospital (Region Syddanmark, IDS-20100044). All of the animal experiments were performed in compliance with the Joslin Diabetes Center Statement for the Use of Animals in Diabetic Research (Boston, Massachusetts, USA) and with approval of the Institutional Animal Care and Use Committee at the Joslin Diabetes Center.

\section{Author contributions}

GLK conceived the project, designed the experiments, and wrote the manuscript. KP wrote the manuscript, and designed and performed most of the experiments. AM generated IRS1 transgenic mice. QL performed the myograph experiments. CRM prepared primary EC and VSMC. PH performed NO production and $\mathrm{Ca}^{2+}$ flux assay in vitro and in vivo. KS, KM, and YM performed FACS experiments. IW, MK, and EM assisted with data analysis and interpretation and critically read the manuscript. DS assisted with the molecular biology experiments. SRP and LMR provided human mammary artery. PLH shared the AKIeNOS plasmid, virus and mice.

\section{Acknowledgments}

The authors are grateful for the expert technical assistance provided by the Diabetes Research Center at the Joslin Diabetes Center. Plasma lipid profiles were measured at the Hormone Assay \& Analytical Services Core of Vanderbilt University (DK59637). This study was supported by a American Diabetes Association 
mentor-based fellowship, NIH grants DK053105 and DK036836, and the Danish Diabetes Academy, which is supported by the Novo Nordisk Foundation. We thank Allen Clermont for performing all mouse metabolic assays at Joslin Diabetes Center. We thank the staffs in the Advanced Genomics and Genetics Core in the Diabetic Research Center at the Joslin Diabetes Center. We also thank M.S. Daad Abraham for her excellent assistance with manuscript preparation. We also thank David Pober for performing statistics with all figures.

Address correspondence to: George L. King, Research Division, Joslin Diabetes Center, One Joslin Place, Boston, Massachusetts 02215, USA. Phone: 617.309.2622; E-mail: George.king@joslin.harvard.edu.

1. Bornfeldt KE, Tabas I. Insulin resistance, hyperglycemia, and atherosclerosis. Cell Metab. 2011;14(5):575-585.

2. Rask-Madsen C, King GL. Vascular complications of diabetes: mechanisms of injury and protective factors. Cell Metab. 2013;17(1):20-33.

3. Steinberg HO, Chaker H, Leaming R, Johnson A, Brechtel G, Baron AD. Obesity/insulin resistance is associated with endothelial dysfunction. J Clin Invest. 1996;97(11):2601-2610.

4. Rask-Madsen C, et al. Loss of insulin signaling in vascular endothelial cells accelerates atherosclerosis in apolipoprotein E null mice. Cell Metab. 2010;11(5):379-389.

5. Tsuchiya $\mathrm{K}$, et al. FoxOs integrate pleiotropic actions of insulin in vascular endothelium to protect mice from atherosclerosis. Cell Metab. 2012;15(3):372-381.

6. Fernandez-Hernando C, et al. Loss of Akt1 leads to severe atherosclerosis and occlusive coronary artery disease. Cell Metab. 2007;6(6):446-457.

7. Jiang ZY, et al. Characterization of selective resistance to insulin signaling in the vasculature of obese Zucker (fa/fa) rats. J Clin Invest. 1999;104(4):447-457.

8. Fulton D, et al. Regulation of endothelium-derived nitric oxide production by the protein kinase Akt. Nature. 1999;399(6736):597-601.

9. Dimmeler S, Fleming I, Fisslthaler B, Hermann C, Busse R, Zeiher AM. Activation of nitric oxide synthase in endothelial cells by Akt-dependent phosphorylation. Nature. 1999;399(6736):601-605.

10. Zeng G, Quon MJ. Insulin-stimulated production of nitric oxide is inhibited by wortmannin. J Clin Invest. 1996;98(4):894-898.

11. Zeng G, et al. Roles for insulin receptor, PI3-kinase, and Akt in insulin-signaling pathways related to production of nitric oxide in human vascular endothelial cells. Circulation. 2000;101(13):1539-1545.

12. Kuhlencordt PJ, et al. Accelerated atherosclerosis, aortic aneurysm formation, and ischemic heart disease in apolipoprotein E/ endothelial nitric oxide synthase double-knockout mice. Circulation. 2001;104(4):448-454.

13. Despres JP, et al. Hyperinsulinemia as an independent risk factor for ischemic heart disease. N Engl J Med. 1996;334(15):952-957.

14. Pyorala K. Relationship of glucose tolerance and plasma insulin to the incidence of coronary heart disease: results from two population studies in Finland. Diabetes Care. 1979;2(2):131-141.

15. Kuboki K, et al. Regulation of endothelial constitutive nitric oxide synthase gene expression in endothelial cells and in vivo: a specific vascular action of insulin. Circulation. 2000;101(6):676-681.

16. Naruse K, et al. Activation of vascular protein kinase C- $\beta$ inhibits Akt-dependent endothelial nitric oxide synthase function in obesity-associated insulin resistance. Diabetes. 2006;55(3):691-698.

17. Rask-Madsen C, et al. Hyperinsulinemia does not change atherosclerosis development in apolipoprotein E null mice. Arterioscler Thromb Vasc Biol. 2012;32(5):1124-1131.

18. Ozaki M, et al. Overexpression of endothelial nitric oxide synthase accelerates atherosclerotic lesion formation in apoE-deficient mice. J Clin Invest. 2002;110(3):331-340.

19. Cybulsky MI, et al. A major role for VCAM-1, but not ICAM-1, in early atherosclerosis. J Clin Invest. 2001;107(10):1255-1262.

20. Ley K, Huo Y. VCAM-1 is critical in atherosclerosis. J Clin Invest. 2001;107(10):1209-1210.

21. Cybulsky MI, Gimbrone MA Jr. Endothelial expression of a mononuclear leukocyte adhesion molecule during atherogenesis. Science. 1991;251(4995):788-791.

22. Khan BV, Parthasarathy SS, Alexander RW, Medford RM. Modified low density lipoprotein and its constituents augment cytokine-activated vascular cell adhesion molecule-1 gene expression in human vascular endothelial cells. J Clin Invest. 1995;95(3):1262-1270.

23. Eguchi S, Hirata Y, Imai T, Marumo F. Endothelin-1 as an autocrine growth factor for endothelial cells. J Cardiovasc Pharmacol. 1995;26(suppl 3):S279-S283.

24. Dagassan PH, et al. Up-regulation of endothelin-B receptors in atherosclerotic human coronary arteries. J Cardiovasc Pharmacol. 1996;27(1):147-153.

25. D'Orleans-Juste P, Labonte J, Bkaily G, Choufani S, Plante M, Honore JC. Function of the endothelin(B) receptor in cardiovascular physiology and pathophysiology. Pharmacol Ther. 2002;95(3):221-238.

26. Park JY, et al. Induction of endothelin-1 expression by glucose: an effect of protein kinase C activation. Diabetes. 2000;49(7):1239-1248.

27. Jiang ZY, et al. Endothelin-1 modulates insulin signaling through phosphatidylinositol 3-kinase pathway in vascular smooth muscle cells. Diabetes. 1999;48(5):1120-1130.

28. Ishikawa $\mathrm{K}$, et al. Biochemical and pharmacological profile of a potent and selective endothelin B-receptor antagonist, BQ-788. Proc Natl Acad Sci U S A. 1994;91(11):4892-4896.

29. Chu TS, Peng Y, Cano A, Yanagisawa M, Alpern RJ. Endothelin(B) receptor activates NHE-3 by a Ca ${ }^{2+}$-dependent pathway in OKP cells. J Clin Invest. 1996;97(6):1454-1462.

30. Hirata Y, et al. Endothelin receptor subtype B mediates synthesis of nitric oxide by cultured bovine endothelial cells. J Clin 
Invest. 1993;91(4):1367-1373.

31. Deliu E, et al. Intracellular endothelin type B receptor-driven $\mathrm{Ca}^{2+}$ signal elicits nitric oxide production in endothelial cells. $J$ Biol Chem. 2012;287(49):41023-41031.

32. Kashiwagi S, et al. eNOS phosphorylation on serine 1176 affects insulin sensitivity and adiposity. Biochem Biophys Res Commun. 2013;431(2):284-290.

33. Terasaka N, et al. ATP-binding cassette transporter G1 and high-density lipoprotein promote endothelial NO synthesis through a decrease in the interaction of caveolin-1 and endothelial NO synthase. Arterioscler Thromb Vasc Biol. 2010;30(11):2219-2225.

34. Kubota T, et al. Impaired insulin signaling in endothelial cells reduces insulin-induced glucose uptake by skeletal muscle. Cell Metab. 2011;13(3):294-307.

35. Kubota T, et al. Lack of insulin receptor substrate-2 causes progressive neointima formation in response to vessel injury. Circulation. 2003;107(24):3073-3080.

36. Yanagisawa M, Kurihara H, Kimura S, Goto K, Masaki T. A novel peptide vasoconstrictor, endothelin, is produced by vascular endothelium and modulates smooth muscle $\mathrm{Ca}^{2+}$ channels. J Hypertens Suppl. 1988;6(4):S188-191.

37. Tirapelli CR, et al. Functional characterization and expression of endothelin receptors in rat carotid artery: involvement of nitric oxide, a vasodilator prostanoid and the opening of $\mathrm{K}^{+}$channels in ETB-induced relaxation. BrJ Pharmacol. 2005;146(6):903-912.

38. Luscher TF, Barton M. Endothelins and endothelin receptor antagonists: therapeutic considerations for a novel class of cardiovascular drugs. Circulation. 2000;102(19):2434-2440.

39. Ohashi Y, et al. Hypotension and reduced nitric oxide-elicited vasorelaxation in transgenic mice overexpressing endothelial nitric oxide synthase. J Clin Invest. 1998;102(12):2061-2071.

40. Teppala S, Shankar A. Association between serum IGF-1 and diabetes among U. Diabetes Care. 2010;33(10):2257-2259.

41. Stuart JA, Page MM. Plasma IGF-1 is negatively correlated with body mass in a comparison of 36 mammalian species. Mech Ageing Dev. 2010;131(9):591-598.

42. Sandhu MS, Heald AH, Gibson JM, Cruickshank JK, Dunger DB, Wareham NJ. Circulating concentrations of insulin-like growth factor-I and development of glucose intolerance: a prospective observational study. Lancet. 2002;359(9319):1740-1745

43. Ansar S, et al. MAPK signaling pathway regulates cerebrovascular receptor expression in human cerebral arteries. BMC Neurosci. 2013;14:12.

44. Li Q, et al. Induction of vascular insulin resistance and endothelin-1 expression and acceleration of atherosclerosis by the overexpression of protein kinase C-beta isoform in the endothelium. Circ Res. 2013;113(4):418-427.

45. Schmittgen TD, Livak KJ. Analyzing real-time PCR data by the comparative C(T) method. Nat Protoc. 2008;3(6):1101-1108. 